\title{
Vortex Trajectory of Rotating Shaft Undergoing Vibration Coupling of Parallel Multicylinders
}

\author{
Ben Zhang, ${ }^{1}$ Yu Li, ${ }^{1}$ Zhong Tang $\mathbb{D}^{1}{ }^{1}$ Hao Zhang, ${ }^{1}$ Yaquan Liang, and Zhan Su${ }^{2}$ \\ ${ }^{1}$ Key Laboratory of Modern Agricultural Equipment and Technology of Ministry of Education, Jiangsu University, Zhenjiang, \\ Jiangsu 212013, China \\ ${ }^{2}$ Key Laboratory of Crop Harvesting Equipment Technology of Zhejiang Province, Jinhua, Zhejiang 321017, China \\ Correspondence should be addressed to Zhong Tang; tangzhong2012@126.com
}

Received 4 October 2021; Accepted 24 December 2021; Published 22 January 2022

Academic Editor: Georgios I. Giannopoulos

Copyright ( $\odot 2022$ Ben Zhang et al. This is an open access article distributed under the Creative Commons Attribution License, which permits unrestricted use, distribution, and reproduction in any medium, provided the original work is properly cited.

\begin{abstract}
Multiple threshing cylinders of combine harvester are mainly driven in parallel, whose transmission methods are complex causing the excitations coupled with each other. The mutual excitation coupling of multiple threshing cylinders causes a severe vibration state of the combine harvester with poor stability. In order to analyze the influence of transmission system coupling on the unbalanced response of the parallel multicylinder system, this paper established a mathematical model of the parallel multicylinder system and derived the general vortex trajectory expression of the unbalanced response of threshing cylinder shaft undergoing vibration coupling of parallel multicylinders. Vibration tests of the bearing seat and the rotating shaft were carried out for comparison and verification. Results showed that the existence of the transmission chain and the transmission mode cause the boundary conditions of the multicylinder system to change, which ultimately led to changes in the unbalanced response of the transmission system. The contribution rate of different transmission methods and rotation speeds to the vibration amount at the fundamental frequency of the multicylinder was $5 \%$ to $30 \%$. The existence of the transmission system caused the vibrations of the various units to be coupled with each other, mutually transmitted, and superimposed, leading to changes in the vortex shape of the rotating shaft and the unbalanced response of the transmission system.
\end{abstract}

\section{Introduction}

As complex agricultural machinery, a combined harvester can complete crop harvesting, threshing, separation, cleaning, and other operations at one time, which is of great significance for improving production efficiency [1-3]. However, the existence of the transmission system and the complexity of transmission methods cause an unbalanced response of the parallel multicylinder system to be coupled and superimposed with each other, resulting in greater vibration and poor stability of the whole machine $[4,5]$. Therefore, it is particularly important to explore the influence mechanism of the transmission system coupling on the unbalanced response of the multicylinder system.

For the rotor system or transmission system research, the transmission system should be analyzed first. The power transmission method of the combine harvester is belt drive or chain drive, which were researched early. Yoshida et al. [6] proposed a stiffness damping model that can simulate the dynamic characteristics of the belt drive according to the movement characteristics of the toothed belt. Ward et al. [7] took the automobile chain drive system as the research object and conducted theoretical and experimental analysis on the damage mechanism in the working process. Due to the polygon effect in the transmission process of the transmission chain, its vibration state is more complicated [8]. Aiming at the problem of roller chain tooth profile asymmetry, Wang et al. [9] proposed a new type of adjustment method, which effectively reduced the impact effect of the chain in the working process. Regarding the mathematical modeling of the transmission chain, Cepon et al. $[10,11]$ established the mathematical model of the belt drive system based on the absolute nodal coordinate formula. On this basis, the damping mechanism was brought into the 
stiffness and then the damping matrix of the numeric system was solved. These results showed that the numerical results were in good agreement with the experimental results. In order to further improve the mathematical model of the roller chain, Pereira et al. [12] used the method of continuous contact force to further describe the contact phenomenon in the mathematical model of the roller chain about the clearance movement problem.

As multirotor systems, mathematical modeling and numerical solution analysis are important means to reveal the influence of the system's vibration response. The general dynamic model of the rolling bearing rotor system was proposed by Li et al. [13], which seamlessly coupled the explicit finite element model (EFEM) of the rotor with the dynamic bearing model (DBM). A finite element modeling, analysis, and identification method for a fully suspended flexible rotor fault based on active magnetic bearings was studied by Kumar et al. [14]. There are many research studies on the mathematical modeling of multirotor systems. With the particularity and complexity of the objects, more and more modeling methods for specific objects have been researched and developed. Filsoof et al. [15] proposed a novel method and system modal dynamic modeling method for multirotor wind turbines; Mereles [16] proposed a method based on distributed parameters or continuous models; these proposed methods can be applied to complex rotor systems with multistep sections and multidisks and multibearings. In order to solve specific eigenvalues, some scholars have proposed a new method for modeling complex rotor systems with multiple discs and multiple bearingscontinuous segmentation method [17]. With the increasing complexity of research objects, the solving methods for specific mathematical models have irreplaceable superiority for studying system dynamics and revealing the nature of system influence relations.

Dynamic characteristics of the rotor system are extremely important for revealing the law of influence among the various working parts of the system. Dupont $[18,19]$ studied the effect of shaft speed and temperature on bearing clearance. Elijah et al. [20] established a dual-rotor mathematical model considering the friction coefficient and eccentricity between the stator and the rotor. The solution results of its dynamic characteristics were expressed and processed through three-dimensional bifurcation diagrams, Poincaré diagrams, and spectral intensity diagrams. The dynamic behavior of the bolted joints at the transition points of different tangential stiffness and bending stiffness was analyzed by Li et al. [21]. A mathematical model of the dynamic characteristics of the rotor-bearing-coupled system under the influence of flexibility, radial clearance, dynamic and static friction, raceway defects, and surface ripples was established by Cheng et al. [22]. The bifurcation diagram was used to analyze the influence of typical parameters on the mechanical characteristics and nonlinear dynamic response of the rotor system. For the dynamic characteristics of the parallel multirotor system, the influence of its internal transmission system is also an important factor that cannot be ignored.
The coupling of the transmission system will cause a large change in the vibration response of the system. Taking 1 to 3 discs as an example, the natural frequency and mode shape of the system were calculated by Chui et al. [23]. Zhang et al. [24] proposed a new data flow analysis model (PN-PDDP), which effectively solved the problem of information complexity in the design process of complex multirotor coupling systems. Existing research mainly focuses on the coupling characteristics of the gear-rotor system. The above researches are mainly based on the gear transmission system, and its influence on the vibration response of the system has been tested and theoretically analyzed. However, there is less research on the influence of chain or belt transmission on the vibration characteristics of the system. This paper revealed the nature of the influence of the coupling of the transmission system on the motion state of the shaft of the parallel multicylinder system from the perspective of theoretical analysis and experimental comparison.

\section{Materials and Methods}

2.1. Mathematical Modeling of Parallel Multicylinders. A combine harvester is a complex transmission system, which is a chain or belt transmission. Multiple components of the combine harvester, such as the engine, the conveying tank, the threshing cylinders, and the fan, are all transmitted through the chain. The power transmission system and transmission mode of the combine harvester are shown in Figure 1. The unbalanced vibration of these rotating parts will cause serious vibration of the whole machine to affect its stability $[25,26]$. In addition, the mutual coupling between the rotating bodies will also cause the unbalanced response in the system to be more complicated [27].

In order to establish the mathematical model of unilateral transmission through the lumped parameter method, the chain drive was simplified as a parallel system of multiple springs and dampers in this article. The relative position of adjacent nodes can be equivalent to a cantilever beam structure. So, the relationship between the displacement and the force between the nodes was established. The calculation model is shown in Figure 2.

The vibration equation of three-cylinder unilateral transmission can be calculated through the lumped parameter method as

$$
[M]\{\ddot{q}\}+\left[D^{\prime}\right]\{\dot{q}\}+\left(\left[\mathrm{K}^{\prime}\right]+[K]\right)\{q\}=\left\{\omega^{2} F_{q}\right\},
$$

where $[M]$ is the mass matrix; $\left[D^{\prime}\right]$ is the damping matrix composed of the damping of the support unit and the transmission system and the coupling coefficient between them; $\left[K^{\prime}\right]$ is the coupling stiffness matrix of the system; $[\mathrm{K}]$ is the stiffness matrix of each rotating shaft in the system; $\{q\},\{\dot{q}\}$, and $\{\ddot{q}\}$ are the displacement vector, velocity vector, and acceleration vector of each node in the system; and $\left\{\omega^{2} F_{q}\right\}$ is the excitation force vector composed of the unbalanced excitation force acting on each unit of the system. 


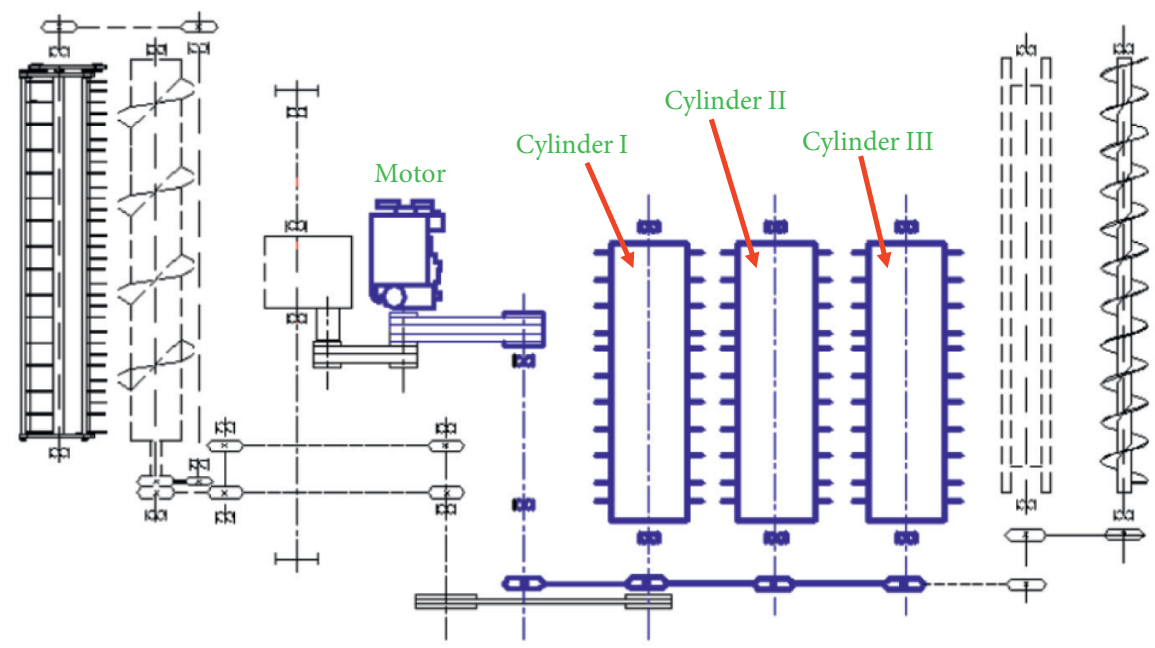

Figure 1: Multicylinder power transmission route belonging to combine harvester.

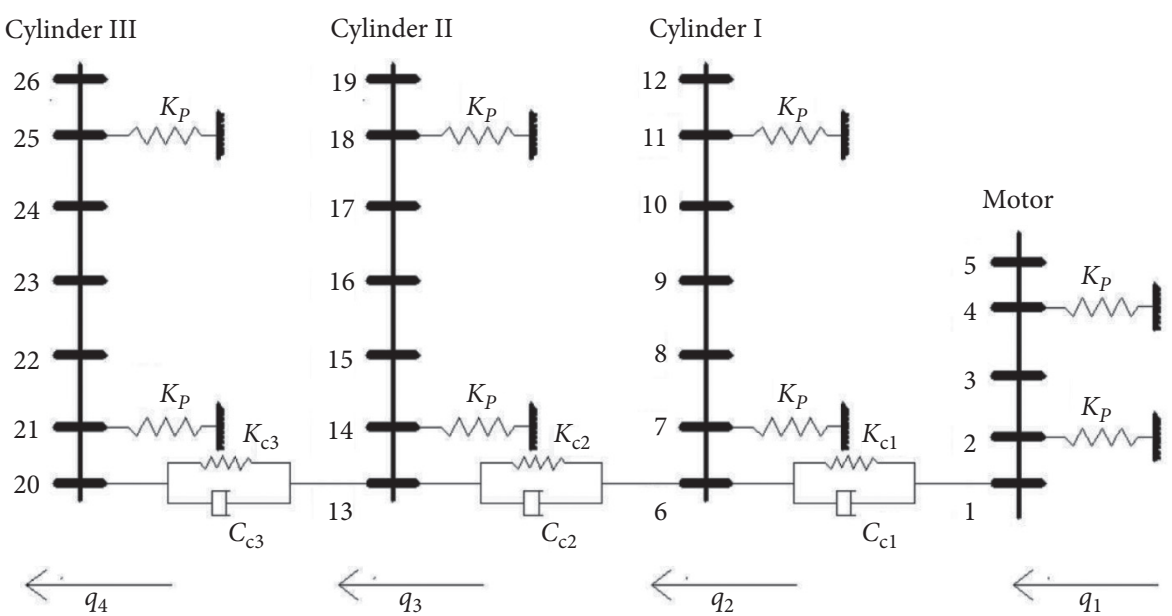

FIGURE 2: Dynamic calculation model of the multicylinder.

In this paper, the general solution of the system equation was deduced by the modal superposition method. On this basis, it was solved by programming. By solving this equation, the influence of the coupling of the transmission system on the unbalanced response of each unit in the system was analyzed.

\subsection{Coupling Unbalances Response of Parallel Multicylinders}

\subsubsection{Unbalanced Response of the Single Threshing Cylinder.} For a single threshing cylinder-bearing system, when the lumped discs are $n$, it has $n$ degrees of freedom. It can be seen that the corresponding equation $[A]\{r\}=w[B]\{r\}$ has $2 n$ eigenvalues and eigenvectors. In general, the rack bar of the horizontal axial flow threshing cylinder is lighter in weight and relatively short in length. Its working speed is generally $800 \mathrm{r} / \mathrm{min}$. Therefore, regardless of the influence of the gyro torque, the mass matrix, stiffness matrix, and damping matrix of a single threshing cylinder are all diagonal matrices. It can be seen that the solved $2 n$ eigenvalues are complex numbers that are conjugate to each other. The corresponding $2 n$ eigenvectors are also conjugate. So, the left modal matrix $[L]$ and the right modal matrix $[R]$ of parallel multicylinder in the modal coordinate system can be established. Then, the expression form of the two modal matrices is as follows: $[L]=\left[l^{(1)}, l^{(2)}, \ldots, l^{(n)}, \bar{l}^{(1)}, \bar{l}^{(2)}\right.$, $\left.\ldots, \bar{l}^{(n)}\right] ;[R]=\left[r^{(1)}, r^{(2)}, \ldots, r^{(n)}, \bar{r}^{(1)}, \bar{r}^{(2)}, \ldots, \bar{r}^{(n)}\right]$.

It can be obtained from the matrix equation of the parallel multicylinder that

$$
\begin{aligned}
& {[L]^{T}[A][R]=[I],} \\
& {[L]^{T}[B][R]=\operatorname{diag}\left[w_{1}, w_{2}, \ldots, w_{n}, \bar{w}_{1}, \bar{w}_{2}, \ldots, \bar{w}_{n}\right] .}
\end{aligned}
$$

Let the horizontal and vertical displacement vectors of each lumped disc of the threshing cylinder be $\{X\}$ and $\{Y\}$, respectively. Each lumped disc can be equivalent to a mass point or node. Assuming that there are $n$ nodes in the threshing cylinder-bearing system, the vector $\{q\}$ formed by the displacement of each node can be expressed as follows: 


$$
\{q\}=\left\{X^{T}, Y^{T}\right\}^{T}=\left\{x_{1}, x_{2} \ldots x_{n}, y_{1}, y_{2} \ldots y_{n}\right\}^{T}
$$

The motion equation of the system can be expressed as follows:

$$
[M]\{\ddot{q}\}+[D]\{\dot{q}\}+[K]\{q\}=\Omega^{2}\{F\},
$$

where $[M],[D]$, and $[K]$ are the mass, damping, and stiffness matrices of the threshing cylinder-bearing system, respectively.

Then, the external force $\{F\}$ can be decomposed into horizontal and vertical excitation forces. The magnitude of the force is mainly related to the rotation speed, the magnitude of the unbalanced mass, the phase, and the time. Its vector expressions in the horizontal and vertical directions are as follows:

$$
\{F\}=\left\{F_{x}^{T}, F_{y}^{T}\right\}^{T}
$$

where

$$
\begin{aligned}
& \left\{F_{x}\right\}=\left\{m_{1} e_{1} \cos \left(\Omega t+\beta_{1}\right), \ldots m_{n} e_{n} \cos \left(\Omega t+\beta_{n}\right)\right\} \\
& \left\{F_{y}\right\}=\left\{m_{1} e_{1} \sin \left(\Omega t+\beta_{1}\right), \ldots m_{n} e_{n} \sin \left(\Omega t+\beta_{n}\right)\right\} .
\end{aligned}
$$

Transforming the motion equation of the system into the state space is as follows:

$$
[B]\{\dot{p}\}-[A]\{p\}=\Omega^{2}\left\{F^{\prime}\right\},
$$

where $[A]=\left[\begin{array}{cc}-K & \\ & M\end{array}\right] ;[B]=\left[\begin{array}{cc}D & M \\ M & 0\end{array}\right] ;\left\{F^{\prime}\right\}=\left\{F^{T}, 0\right\}^{T}$; and $\{p\}=\left\{q^{T}, \dot{q}^{T}\right\}^{T}$.

The state coordinate is decomposed into the product of the matrix formed by the right eigenvector of the threshing cylinder system and the modal coordinate.

$$
\{p\}=[R]\{\eta\},
$$

where $\{\eta\}$ is canonical coordinates; $[R]$ is a matrix composed of the right eigenvectors of the threshing cylinder system; $\{p\}$ is the state coordinate of the threshing cylinder-bearing system which is the vector used to characterize the motion state of each node in the system.

Based on Equation (7), (8) is as follows:

$$
[B][R]\{\dot{\eta}\}-[A][R]\{\eta\}=\Omega^{2}\left\{F^{\prime}\right\} .
$$

Multiplying both sides of the equation by the left eigenvector of the entire system at the same time $[L]^{T}$, equation (9) is as follows:

$$
[L]^{T}[B][R]\{\dot{\eta}\}-[L]^{T}[A][R]\{\eta\}=\Omega^{2}\left\{T^{\prime}\right\},
$$

where $\Omega^{2}\left\{T^{\prime}\right\}$ is the generalized excitation force of the threshing cylinder due to dynamic imbalance:

$$
\begin{aligned}
\left\{T^{\prime}\right\} & =[L]^{T}\left\{F^{\prime}\right\}=\left\{T^{T}, \bar{T}^{T}\right\}^{T}, \\
\{T\} & =\left\{t_{1}, t_{2}, \ldots t_{n}\right\}^{T} .
\end{aligned}
$$

By

$$
\begin{aligned}
& {[L]^{T}[A][R]=[I],} \\
& {[L]^{T}[B][R]=\operatorname{diag}\left[w_{1}, w_{2}, \ldots, w_{n}, \bar{w}_{1}, \bar{w}_{2}, \ldots, \bar{w}_{n}\right] .}
\end{aligned}
$$

It can be seen that by bringing it into the equation, it can be decomposed into " $2 \mathrm{n}$ " equations represented by canonical coordinates in the modal space, namely,

$$
\left\{\begin{array}{c}
\dot{\eta}_{1}-w_{1}^{2} \eta_{1}=\Omega^{2} t_{1}, \\
\dot{\eta}_{2}-w_{2}^{2} \eta_{2}=\Omega^{2} t_{2}, \\
\cdot \\
\cdot \\
\dot{\eta}_{n}-w_{n}^{2} \eta_{n}=\Omega^{2} t_{n}, \\
\dot{\eta}_{n+1}-\bar{w}_{1}^{2} \eta_{n+1}=\Omega^{2} \bar{t}_{1}, \\
\dot{\eta}_{n+2}-\bar{w}_{2}^{2} \eta_{n+2}=\Omega^{2} \bar{t}_{2}, \\
\cdot \\
\cdot \\
\dot{\eta}_{n+n}-\bar{w}_{n}^{2} \eta_{n+n}=\Omega^{2} \bar{t}_{n} .
\end{array}\right.
$$

As shown in equation (11), it can be seen that when the threshing cylinder-bearing system has damping, its unbalanced response can be converted to the response under the excitation of the generalized excitation force $\Omega^{2}\left\{T^{\prime}\right\}$ in the canonical coordinate system. This realizes the decoupling between the motion nodes in the modal space. The proportion of each mode in the unbalanced response under the specific exciting force can be obtained.

According to the expression of exciting force, $\Omega^{2} t_{i}$ can be expressed as follows:

$$
\Omega^{2} t_{i}=\Omega^{2}\left(t_{c i} \cos \Omega t+t_{s i} \sin \Omega t\right) .
$$

Then, the $i$-th equation can be expressed as follows:

$$
\dot{\eta}_{i}-w_{i}^{2} \eta_{i}=\Omega^{2}\left(t_{c i} \cos \Omega t+t_{s i} \sin \Omega t\right),
$$

where $t_{c i}$ and $t_{s i}$ are plural.

The particular solution of this equation is $\eta_{i}=A_{i} \cos \Omega \mathrm{t}+B_{i} \sin \Omega \mathrm{t}$, bringing it in

$$
\begin{aligned}
& A_{i}=-\Omega^{2} \frac{\left(\Omega t_{s i}+w_{i} t_{c i}\right)}{w_{i}^{2}+\Omega^{2}}, \\
& B_{i}=\Omega^{2} \frac{\left(\Omega t_{c i}-w_{i} t_{s i}\right)}{w_{i}^{2}+\Omega^{2}},
\end{aligned}
$$

then 
$\eta_{i}=\frac{\Omega^{2}}{w_{i}^{2}+\Omega^{2}}\left(-\left(\Omega t_{s i}+w_{i} t_{c i}\right) \cos \Omega t+\left(\Omega t_{c i}-w_{i} t_{s i}\right) \sin \Omega t\right)$.

As shown in equation (11), we know that $\eta_{n+i}=\bar{\eta}_{i}$. It can be obtained that the unbalanced response of the threshing cylinder system under the action of the unbalanced excitation force $\Omega^{2}\left\{F^{\prime}\right\}$ under the physical coordinates $\{p\}$ is as follows:

$$
\{p\}=[R]\{\eta\}=\sum_{i=1}^{2 n} \eta_{i}\left\{r^{(i)}\right\}=\sum_{i=1}^{n} \eta_{i}\left\{r^{(i)}\right\}+\bar{\eta}_{i}\left\{\bar{r}^{(i)}\right\} .
$$

The unbalanced response $\{p\}$ includes the vibration response of each node in the threshing cylinder system in the physical coordinate system. The vibration response of the node numbered $k$ is as follows:

$$
p_{k}=\sum_{i=1}^{n} \eta_{i}\left\{r_{k}^{(i)}\right\}+\bar{\eta}_{i}\left\{\bar{r}_{k}^{(i)}\right\}
$$

It can be obtained that the unbalanced response of each node in the threshing cylinder under the physical coordinates due to the dynamic unbalance is the result of the superposition of the conjugate mode of the system $n$. The density occupied by each mode, that is, its contribution to the unbalanced response of the node, is related to $\eta_{i}$ in the canonical coordinate system.

\subsubsection{Unbalanced Response of Parallel Multicylinders.} The transmission system is incorporated into the parallel multicylinder system so that the cylinder units are connected together to form a whole. Assuming that there are $m$ parallel threshing cylinders in the parallel multicylinder system, their speeds are $\Omega_{1}, \Omega_{2}, \ldots \Omega_{m}$. The speed of each threshing cylinder is different. Assuming that the parallel multicylinder system has $n$ nodes, the number of nodes on each threshing cylinder is $k_{1}, k_{2}, \ldots k_{m}$, where $n=k_{1}+k_{2}$ $+\ldots+k_{m}$. Sort the unbalanced excitation force on the nodes according to the order of each node to obtain the excitation force vector of the parallel multicylinder system.

$$
\{F\}=\left\{F_{x}^{T}, F_{y}^{T}\right\}^{T},
$$

where

$$
\begin{aligned}
F_{x}= & \left\{m_{1} e_{1} \Omega_{1}^{2} \cos \left(\Omega_{1} t+\beta_{1}\right), \ldots, m_{k_{1}} e_{k_{1}} \Omega_{1}^{2} \cos \left(\Omega_{1} t+\beta_{1}\right), m_{k_{1}+1} e_{k_{1}+1} \Omega_{2}^{2} \cos \left(\Omega_{1} t+\beta_{1}\right), \ldots m_{k_{2}} e_{k_{2}} \Omega_{2}^{2} \cos \left(\Omega_{1} t+\beta_{1}\right),\right. \\
& \left.\ldots m_{k_{m}} e_{k_{m}} \Omega_{m}^{2} \cos \left(\Omega_{m} t+\beta_{1}\right)\right\}, \\
F_{y}= & \left\{m_{1} e_{1} \Omega_{1}^{2} \sin \left(\Omega_{1} t+\beta_{1}\right), \ldots, m_{k_{1}} e_{k_{1}} \Omega_{1}^{2} \sin \left(\Omega_{1} t+\beta_{1}\right), m_{k_{1}+1} e_{k_{1}+1} \Omega_{2}^{2} \sin \left(\Omega_{1} t+\beta_{1}\right), \ldots m_{k_{2}} e_{k_{2}} \Omega_{2}^{2} \sin \left(\Omega_{1} t+\beta_{1}\right),\right. \\
& \left.\ldots m_{k_{m}} e_{k_{m}} \Omega_{m}^{2} \sin \left(\Omega_{m} t+\beta_{1}\right)\right\} .
\end{aligned}
$$

Applying the same method, the equation of motion of the parallel multicylinder system is transformed into the state space to obtain as follows:

$$
[B]\{\dot{p}\}-[A]\{p\}=\Omega^{2}\left\{F^{\prime}\right\} .
$$

Coordinate transformation can be obtained as follows:

$$
[B][R]\{\dot{\eta}\}-[A][R]\{\eta\}=\Omega^{2}\left\{F^{\prime}\right\} .
$$

Multiplying both sides of the formula by the left eigenvector of the entire system at the same time $[L]^{T}$, we get

$$
[L]^{T}[B][R]\{\dot{\eta}\}-[L]^{T}[A][R]\{\eta\}=\Omega^{2}\left\{T^{\prime}\right\},
$$

where $\Omega^{2}\left\{T^{\prime}\right\}$ is the generalized excitation force of the threshing cylinder due to dynamic imbalance.

$$
\left\{T^{\prime}\right\}=[L]^{T}\left\{F^{\prime}\right\}=\left\{T^{T}, \bar{T}^{T}\right\}^{T},
$$

where $\{T\}=\left\{t_{1}, t_{2}, \ldots t_{n}\right\}^{T}$.

Each excitation force element in $t_{-} 1, t \_2, \ldots, t \_n$ contains the excitation component related to the rotation frequency of each threshing cylinder in the parallel threshing cylinder system. At this time, the parallel threshing cylinder system is decoupled from the modal space and written into the form of quantity as follows: 


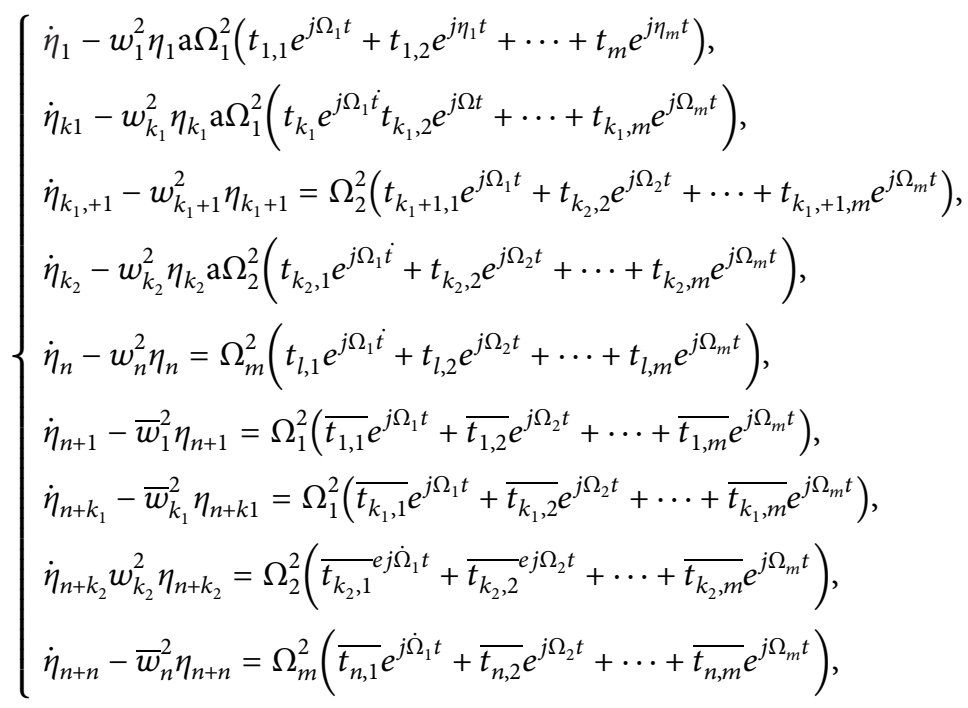

where $t_{r, z}$ is the coefficient of the $r$-th element in the excitation force vector $\{T\}$ before the excitation force related to the $z$ th threshing cylinder. According to the expression of exciting force, $t_{r, z}$ can be expressed as

$$
t_{r, z}=\left(t_{z r}^{c} \cos \Omega t+t_{z r}^{s} \sin \Omega t\right) \text {. }
$$

As shown in equation (26), the exciting force has an effect on each barrel in the parallel barrel system. Therefore, the damped vibration response of each node in the parallel threshing cylinder system under the coupling action of multiple excitation forces can be solved as follows:

$$
\begin{aligned}
p_{i}= & \sum_{r=1}^{k} \sum_{z=1}^{m} \frac{\Omega_{z} t_{z r}^{c}-w_{r}^{2} t_{z r}^{s} \varnothing_{i}^{(r)}}{w_{r}^{2}+\Omega_{z}^{2}} \Omega_{1}^{2}+\sum_{r=1}^{k_{2}} \sum_{z=1}^{m} \frac{\Omega_{z} t_{z r}^{c}-w_{r}^{2} t_{z r}^{s} \varnothing_{i}^{(r)} \Omega_{2}^{2}}{w_{r}^{2}+\Omega_{z}^{2}} \Omega_{2}^{2}+\cdots+\sum_{r=1}^{k_{m}} \sum_{z=1}^{m} \frac{\Omega_{z} t_{z r}^{c}-w_{r}^{2} t_{z r}^{s} \varnothing_{i}^{(r)}}{w_{r}^{2}+\Omega_{z}^{2}} \Omega_{m}^{2} \\
& +\sum_{r=1}^{k_{1}} \sum_{z=1}^{m} \frac{\Omega_{z} t_{z r}^{c}-w_{r}^{2} t_{s z r} \varnothing_{i}^{(r)}}{w_{r}^{2}+\Omega_{z}^{2}} \Omega_{1}^{2}+\sum_{r=1}^{k_{2}} \sum_{z=1}^{m} \frac{\Omega_{z} t_{z r}^{c} w_{r}^{2} t_{z r}^{s} \varnothing_{i}^{(r)}}{w_{r}^{2}+\Omega_{z}^{2}} \Omega_{2}^{2}+\cdots+\sum_{r=1}^{k_{m}} \sum_{z=1}^{m} \frac{\Omega_{z} t_{z r}^{c}-w_{r}^{2} t_{z r}^{s} \varnothing_{i}^{(r)}}{w_{r}^{2}+\Omega_{z}^{2}} \Omega_{m}^{2},
\end{aligned}
$$

where $i$ is the node number; $\rho_{i}$ is the unbalanced response of the $\mathrm{i}$-th node; $\Omega_{z}$ is the speed of each threshing cylinder; $w_{r}$ is the critical speed of each stage of the system; $t_{z r}^{c}$ is the coefficient before " $\cos \Omega \mathrm{t}$ " in the $\mathrm{r}$-th element in the excitation force vector $\{T\}$ and the excitation force related to the $\mathrm{z}$-th threshing cylinder; $t_{z r}^{s}$ is the coefficient before " $\sin \Omega \mathrm{t}$ " in the $\mathrm{r}$-th element in the excitation force vector $\{T\}$ and the excitation force related to the $\mathrm{z}$-th threshing cylinder; $\varnothing_{\mathrm{i}}^{(r)}$ is the element in the $\mathrm{r}$-th column and the i-th row of the matrix $[\varnothing]$ composed of the eigenvectors of the parallel threshing cylinder system.

\subsubsection{Calculation of Unbalanced Response Coupling} Property. During the harvesting process, the threshing cylinder is connected with other working devices through the transmission chain and the frame, causing its working state to be affected by other working parts and the transmission system. Assuming that the frame is in a completely rigid state, the unbalanced response of the threshing cylinder is mainly superimposed and influenced by each other through the transmission system. In order to study the impact of the vibration coupling of the transmission system on the unbalanced response of a single threshing cylinder, this section analyzed the unbalanced response of a single threshing cylinder when it was working alone and the vibration state of the multicylinder system.

In the simulation, the speed of cylinder I was set to " $40 \pi \mathrm{rad} / \mathrm{s}$ ", and an imbalance of 2 " " $\mathrm{kg} \bullet \mathrm{mm}$ " was applied to the No. 7 node of cylinder I. The initial phase of the imbalance was set to the $0^{\circ}$ phase of the system. The support stiffness of the rotating shaft and the damping value of the rolling bearing were $2 \times 10^{7} \mathrm{~N} / \mathrm{m}$ and $200 \mathrm{Ns} / \mathrm{m}$, respectively. The vibration mode at time 0 and the time-domain and frequency-domain characteristics of each node in cylinder I were obtained by MATLAB.

In order to compare the impact of the transmission system on the unbalanced response of cylinder I, cylinder I was connected in parallel with the motor and the other two threshing cylinders unilaterally. The model of the calculation was shown in Figure 2. Before the simulation, the equivalent tensile stiffness of the transmission chain was $188585 \mathrm{~N} / \mathrm{m}$, and the stiffness of the rolling bearing was still $2 \times 10^{7} \mathrm{~N} / \mathrm{m}$. The equivalent damping value of the chain drive was $150 \mathrm{Ns} / \mathrm{m}$. The damping value when the rolling bearing was rotating was $200 \mathrm{Ns} / \mathrm{m}$. During the simulation, the rotational 
speed and unbalanced excitation of cylinder I remained the same as before. The speed of each rotating unit in the system was as follows: the motor speed was " $20 \pi \mathrm{rad} / \mathrm{s}$ "; the cylinder I speed was " $40 \pi \mathrm{rad} / \mathrm{s}$ "; the cylinder II speed was " $60 \pi \mathrm{rad} /$ $\mathrm{s}$ "; the cylinder III speed was " $80 \pi \mathrm{rad} / \mathrm{s}$ ". When calculating, impose an imbalance of $2 \mathrm{~kg} \mathrm{~mm}$ on the No. 7 node of cylinder I; impose an imbalance of 5 " "kg•mm" on the No. 14 node of cylinder II; apply an imbalance of $1 \mathrm{~kg} \bullet \mathrm{mm}$ to the No. 21 node of the cylinder III. After the setting is completed, it was solved by MATLAB.

\subsection{Coupling Characteristic Test of the Parallel Multicylinder System}

2.3.1. Vibration Characteristic Test of the Multicylinder System. In order to study the vibration coupling characteristics and transmission characteristics under different transmission modes, an experimental study was carried out on its proportional test bench. The power transmission system and transmission mode of the combine harvester are shown in Figure 3. Parallel multicylinder included a three-layer structure, with a motor placed on the bottom layer and an eddy current speed sensor installed on the output shaft of the motor. A total of three cylinders with the same structure were placed in the middle layer. Each cylinder has small holes of the same size at both ends to add an unbalanced mass. On this basis, the vibration characteristic of each cylinder under different motion states was analyzed. During the test, the vibration characteristics under three representative working conditions were selected for testing and analysis, which mainly included a single-cylinder vibration characteristic test, three-cylinder unilateral transmission, and three-cylinder bilateral transmission vibration characteristic test. The schematic diagram of the transmission mode of the multicylinder system is shown in Figure 4.

In order to test the excitation sources' impact of the bearing housing, the vibration characteristics of the bearing housing were first tested. Since the position of the bearing seat was the first transmission position of the unbalanced vibration response, the vibration of the bearing seat reflected the vibration characteristics of the unbalanced response on the rotor. In order to analyze the influence of the transmission chain on the motion state of the rotating shaft, a preliminary test and analysis of bearing seat vibration response can be carried out. Then, the displacement signal of the shaft center trajectory of the rotating shaft can be further analyzed and verified.

The vibration test instrument was produced by China Donghua Vibration Test Co., Ltd. The corresponding vibration test acquisition analyzer was DH5902. The vibration test acquisition and analysis system is shown in Figure 5, including a matching computer and a signal acquisition instrument. The vibration test sensor used in the test was a three-axis acceleration sensor produced by Yangzhou Xiyuan Electronic Technology Co., Ltd. The three-axis acceleration sensor for the vibration test is shown in Figure 5.

During the tests, the specific model of the three-axis acceleration sensor, the direction of measuring vibration, and the sensitivity of each direction are shown in Table 1.

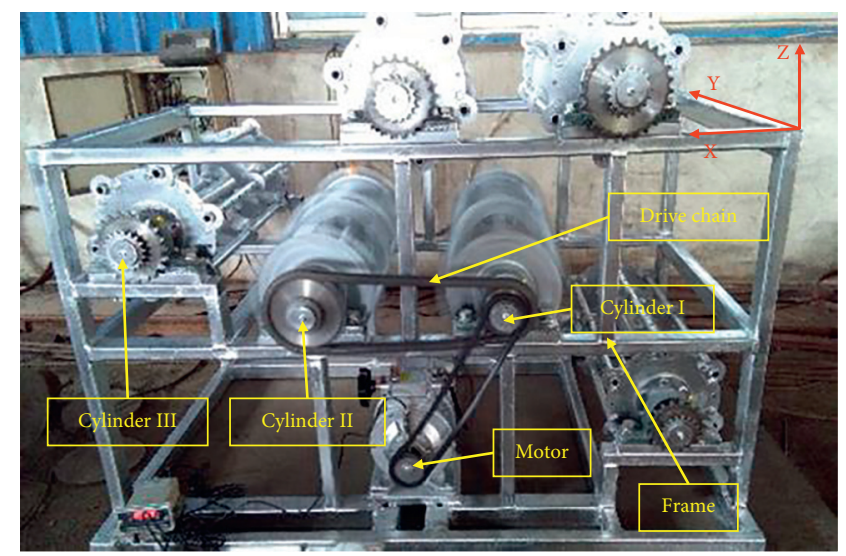

FIGURE 3: Isometric model of the combine harvester parallel multicylinder.

The vibration directions mentioned in this paper were the overall coordinate directions, not the individual sensor directions. The method for testing the vibration characteristics of the single-cylinder bearing housing was to arrange the acceleration sensors 820614 and 820616 on the bearing housings at both ends of the cylinder. The No. 820614 was installed on the bearing housing in the $Y$ direction of the entire system. The No. 820616 was installed on the bearing housing on the other side of the cylinder I. The vibration test method under other transmission modes was the same as this, and the arrangement of the acceleration sensor is shown in Figure 6. There were two main types of vibration characteristics test for a single cylinder. One was to test the vibration characteristics of the bearing seat at different speeds under no load; the other one was to apply an unbalanced mass with a mass of $127.89 \mathrm{~g}$ in its $0^{\circ}$ phase. The method of applying the unbalanced mass in the experiment is shown in Figure 6.

In the vibration experiment, the speed of each cylinder ranges from $600 \mathrm{r} / \mathrm{min}$ to $800 \mathrm{r} / \mathrm{min}$. This section mainly included three working conditions (single-cylinder transmission, three-cylinder unilateral transmission, and three-cylinder bilateral transmission). The single-cylinder drive mode was used as a test reference for the entire system to analyze the impact of the drive system and the dynamic unbalance response of cylinder I.

The main working conditions during the vibration test and the movement state of each cylinder are shown in Table 2.

\subsubsection{Simulation and Experiment of Shaft Wobble Form.} (1) Theoretical vortex shape of threshing cylinder shaft. Suppose the displacement of any node $i$ in the threshing cylinder system is $x_{i}, y_{i}$. According to the solution method of the mode superposition method, the unbalanced response at the node will not bounce in the plane where the original excitation source is located; that is, there is a certain deflection angle between the vibration response and the excitation. This means that there is a certain phase lag in the vibration response in both the horizontal and vertical directions. Therefore, the displacement at node $i$ can be further expressed as follows: 


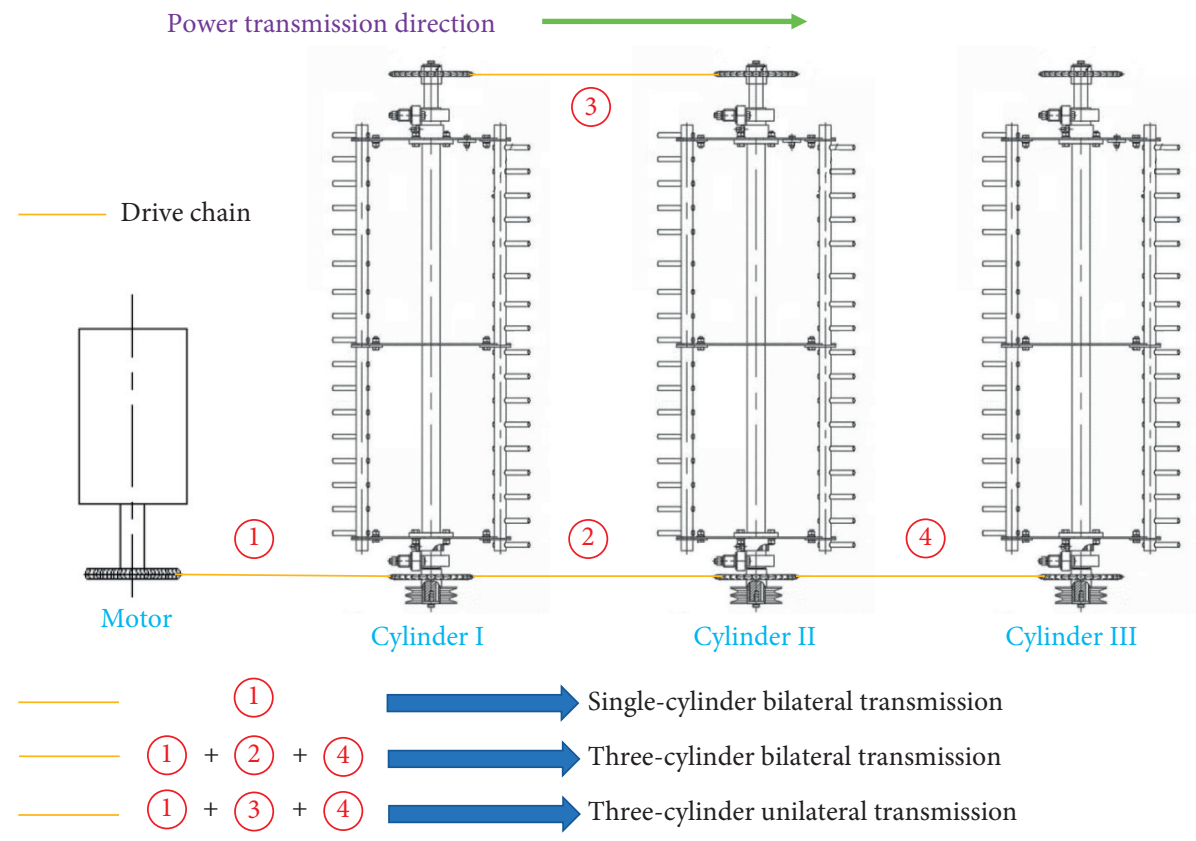

Figure 4: Schematic diagram of multicylinder transmission modes.

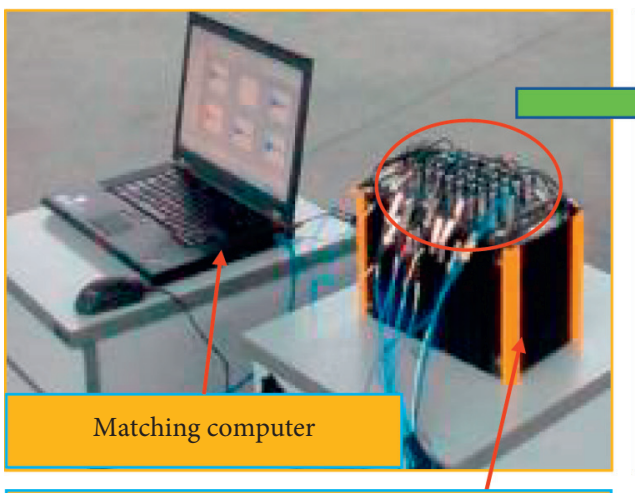

Signal acquisition instrument

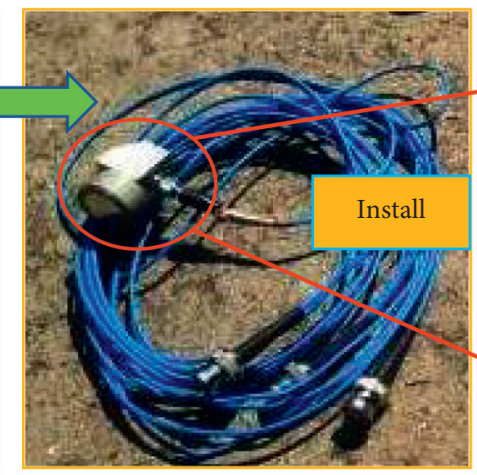

Acceleration sensor

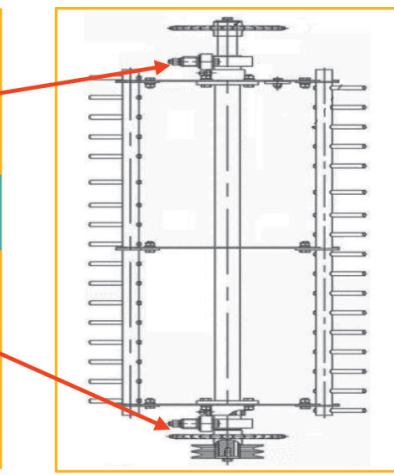

Threshing cylinder

FIGURE 5: Dynamic signal acquisition instrument and three-way acceleration sensor.

TABLE 1: Sensors model and sensitivity in various directions.

\begin{tabular}{|c|c|c|c|c|c|c|c|c|c|}
\hline No. & Model & Direction & Measuring direction & Sensitivity $/ \mathrm{mv} / \mathrm{ms}^{-2}$ & No. & Model & Direction & Measuring direction & Sensitivity $/ \mathrm{mv} / \mathrm{ms}^{-2}$ \\
\hline \multirow{3}{*}{1} & & $\mathrm{X}$ & $\mathrm{X}$ & 1.05 & & & $\mathrm{X}$ & $\mathrm{X}$ & 1.02 \\
\hline & 820614 & $\mathrm{Y}$ & $\mathrm{Y}$ & 1.05 & 4 & 820616 & $\mathrm{Y}$ & $\mathrm{Y}$ & 1.05 \\
\hline & & $\mathrm{Z}$ & $\mathrm{Z}$ & 1.03 & & & $\mathrm{Z}$ & $\mathrm{Z}$ & 1.05 \\
\hline
\end{tabular}

$$
\left\{\begin{array}{l}
x_{i}=x_{c i} \cos (\Omega t)+x_{s i} \sin (\Omega t), \\
y_{i}=y_{c i} \cos (\Omega t)+y_{s i} \sin (\Omega t),
\end{array}\right.
$$

where $x_{c}, x_{s}, y_{c}$, and $y_{s}$ are all real numbers.

In order to study the vortex shape at the internal nodes of the threshing cylinder model, simultaneous analysis of $x$ and $y$ is needed to establish the relationship, so that the vortex shape at the nodes can be solved and analyzed. Therefore, the $x$ and $y$ in equation (29) can be combined together and expressed with a complex number $\rho$.

$$
\rho=x+j y
$$

Then,

$$
\rho=\left(x_{c}+j y_{c}\right) \cos (\Omega t)+\left(x_{s}+j y_{s}\right) \sin (\Omega t) .
$$

From Euler's formula, the above formula can be transformed as follows:

$$
\rho=\rho_{+} e^{j \Omega t}+\rho_{-} e^{-j \Omega t},
$$




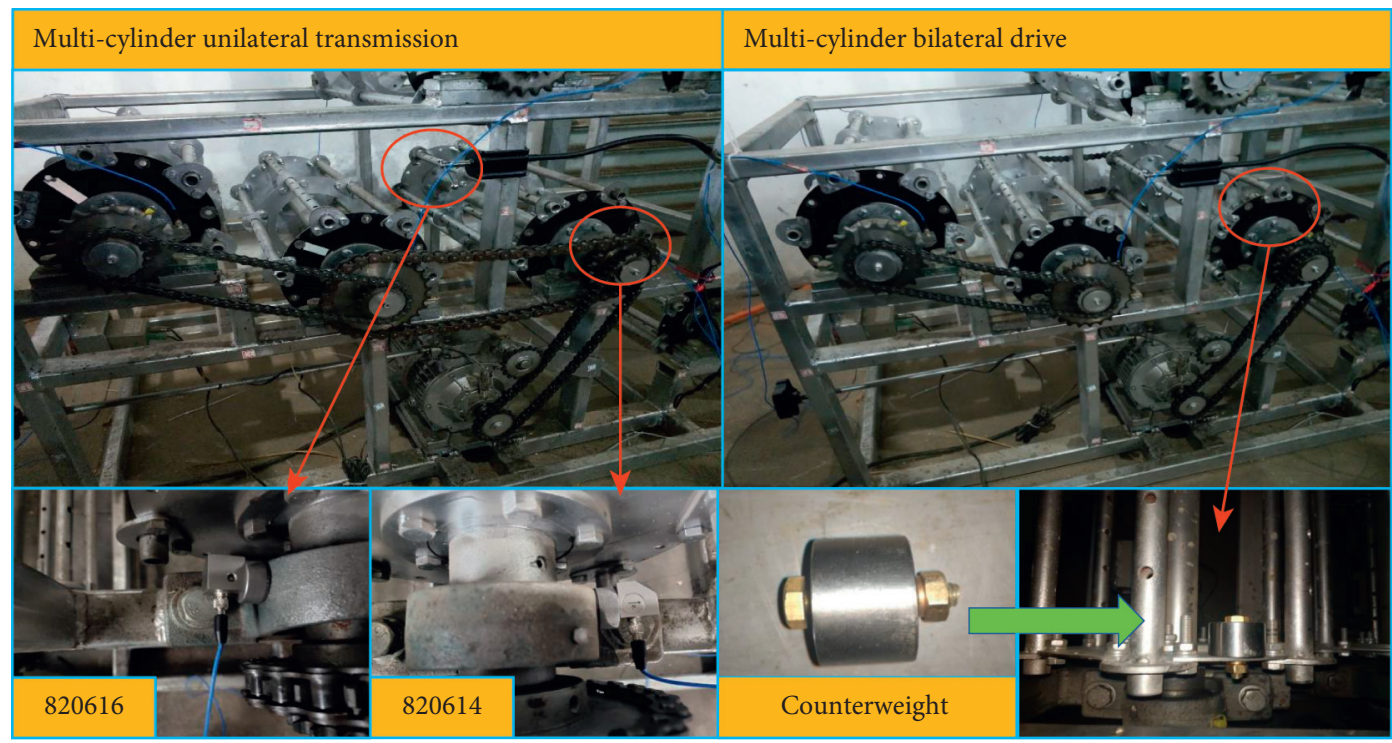

FIgURE 6: Test installation methods under different delivery methods.

TABLE 2: Main working conditions during the test and the movement state of each cylinder.

\begin{tabular}{|c|c|c|c|c|}
\hline Working condition & $\begin{array}{c}\text { Cylinder I } \\
\text { counterweight } \\
(\mathrm{g})\end{array}$ & $\begin{array}{l}\text { Cylinder II counterweight } \\
\text { (g) }\end{array}$ & $\begin{array}{l}\text { Cylinder III counterweight } \\
\text { (g) }\end{array}$ & Motor speed $/ r / \min$ \\
\hline \multirow[t]{2}{*}{ Single-cylinder transmission } & 0 & - & - & \multirow[t]{2}{*}{$\begin{array}{c}600,650,700,750 \\
800\end{array}$} \\
\hline & 127.9 & - & - & \\
\hline \multirow{4}{*}{$\begin{array}{l}\text { Three-cylinder unilateral } \\
\text { transmission }\end{array}$} & 127.89 & 0 & 0 & \multirow[t]{4}{*}{$\begin{array}{c}600,650,700,750, \\
800\end{array}$} \\
\hline & 0 & 227.89 & 0 & \\
\hline & 0 & 0 & 227.89 & \\
\hline & 0 & 0 & 0 & \\
\hline \multirow{4}{*}{$\begin{array}{l}\text { Three-cylinder bilateral } \\
\text { transmission }\end{array}$} & 0 & 0 & 0 & \multirow[t]{4}{*}{$\begin{array}{c}600,650,700,750, \\
800\end{array}$} \\
\hline & 127.89 & 0 & 0 & \\
\hline & 0 & 227.89 & 0 & \\
\hline & 0 & 0 & 227.89 & \\
\hline
\end{tabular}

where $\rho_{+}=\left(\left(\left(x_{c}+y_{s}\right)+j\left(y_{c}-x_{s}\right)\right) / 2\right) ; \quad \rho_{-}=\left(\left(\left(x_{c}-y_{s}\right)\right.\right.$ $\left.\left.+j\left(y_{c}+x_{s}\right)\right) / 2\right)$.

As shown in equation (32), it can be seen that the result described is an ellipse, but the major and minor axes of the ellipse do not completely coincide with $x$ and $y$. The vortex direction, the size, and direction of the major axis and minor axis of this ellipse are closely related to the $x_{c}, x_{s}, y_{c}$, and $y_{s}$ parameters.

Assuming that the rotation angles of $\rho_{+}$and $\rho_{-}$are $\theta_{+}$and $\theta_{-}$, respectively, the expression of the rotation angle can be obtained from equation (31).

$$
\left\{\begin{array}{l}
\operatorname{tg} \theta_{+}=\frac{y_{c}-x_{s}}{x_{c}+y_{s}}, \\
\operatorname{tg} \theta_{-}=\frac{y_{c}+x_{s}}{x_{c}-y_{s}} .
\end{array}\right.
$$

Convert $\rho_{+}$and $\rho_{-}$into exponential form to get

$$
\left\{\begin{array}{l}
\rho_{+}=\left|\rho_{+}\right| e^{j \theta_{+}}, \\
\rho_{-}=\left|\rho_{-}\right| e^{j \theta_{-}} .
\end{array}\right.
$$

Incorporating equations (34) into (32),

$$
\rho=\rho_{1}+\rho_{2}
$$

where

$$
\left\{\begin{array}{l}
\rho_{1}=\left|\rho_{+}\right| e^{j\left(\Omega t+\theta_{+}\right)}, \\
\rho_{2}=\left|\rho_{-}\right| e^{-j\left(\Omega t-\theta_{-}\right)} .
\end{array}\right.
$$

As shown in equation (36), it can be seen that the complex response $\rho$ at any node is formed by the superposition of two complex numbers $\rho_{1}$ and $\rho_{2}$. The trajectory of $\rho_{1}$ is a circular trajectory with a radius of $\left|\rho_{+}\right|$, the angular frequency of its motion is $\Omega$, and its rotation direction is the same as the rotation direction of the shaft. When $t=0$, the initial phase of $\rho_{1}$ is $\theta_{+}$. In the same way, the trajectory of $\rho_{2}$ 
is a circular trajectory with a radius of $\left|\rho_{-}\right|$, and the angular frequency of its motion is $\Omega$, but its rotation direction is opposite to the rotation direction of the shaft. When $t=0$, the initial phase of $\rho_{2}$ is $\theta_{-}$. As shown in Figure 7, it is a schematic diagram of two circular motion trajectories represented by $\rho_{1}$ and $\rho_{2}$. A schematic diagram of the elliptical motion trajectory obtained after the two are superimposed on each other is shown in Figure 8.

Starting from $t=0$, the two circular trajectories move in opposite directions. Supposing the phase angle of $\rho_{1}$ during the movement is $\theta_{1}$, the phase angle during the movement can be expressed as $\theta_{1}=\Omega t+\theta_{+}$. Similarly, suppose the phase angle of $\rho_{2}$ in the movement process is $\theta_{2}=-\Omega t+\theta_{-}$. When they are on the same straight line and their phase angle difference is 0 at a certain time $t$, the position of $\rho_{-} 1$ and $\rho \_2$ is the position of the long axis of the ellipse. At this time, the following conditions are met.

$$
\theta_{1}-\theta_{2}=2 \Omega t+\theta_{+}-\theta_{-}=0
$$

According to equation (31), (36) can be rewritten as follows:

$$
\operatorname{tg} 2 \alpha=\operatorname{tg}\left(\theta_{1}+\theta_{2}\right)=\frac{2\left(x_{c} y_{c}+x_{s} y_{s}\right)}{\left(x_{c}^{2}+x_{s}^{2}\right)-\left(y_{c}^{2}+y_{s}^{2}\right)} .
$$

Let the long axis corresponding to the motion trajectory at the node be $a$, and let the short axis be $b$. From the above analysis, we can get

$$
\left\{\begin{array}{l}
a=\left|\rho_{+}\right|+\left|\rho_{-}\right|, \\
b=\left|\rho_{+}\right|-\left|\rho_{-}\right|,
\end{array}\right.
$$

where

$$
\left\{\begin{array}{l}
\left|\rho_{+}\right|=\frac{1}{2} \sqrt{\left(x_{c}^{2}+x_{s}^{2}\right)+\left(y_{c}^{2}+y_{s}^{2}\right)+2\left(x_{c} y_{s}-x_{s} y_{c}\right)}, \\
\left|\rho_{-}\right|=\frac{1}{2} \sqrt{\left(x_{c}^{2}+x_{s}^{2}\right)+\left(y_{c}^{2}+y_{s}^{2}\right)-2\left(x_{c} y_{s}-x_{s} y_{c}\right)} .
\end{array}\right.
$$

When $\left|\rho_{+}\right|>\left|\rho_{-}\right|$, the corresponding node in the threshing cylinder model makes a positive vortex. On the contrary, when $\left|\rho_{+}\right|<\left|\rho_{-}\right|$, the corresponding nodes in the threshing cylinder model make reverse vortexing.

Based on the above analysis results, it can be seen that during the movement of the threshing cylinder, the movement trajectory of the nodes in the mathematical model is an elliptical movement with different eccentricities and different directions of the long and short axes. The complex displacement of each node has a different phase deflection angle, which causes the vortex trajectory of the axis of the entire system to be a distorted space curve. In addition, the shape of the axis vortex changes every moment and has a certain periodicity. Its angular velocity in the whirling process also changes at every moment, but the time of its whirl cycle is fixed.

(2) Axis trajectory test method of threshing cylinder shaft: in this experiment, the axis trajectory measurement method commonly used in engineering is used to test the state of the rotating shaft. The axis trajectory of the rotating

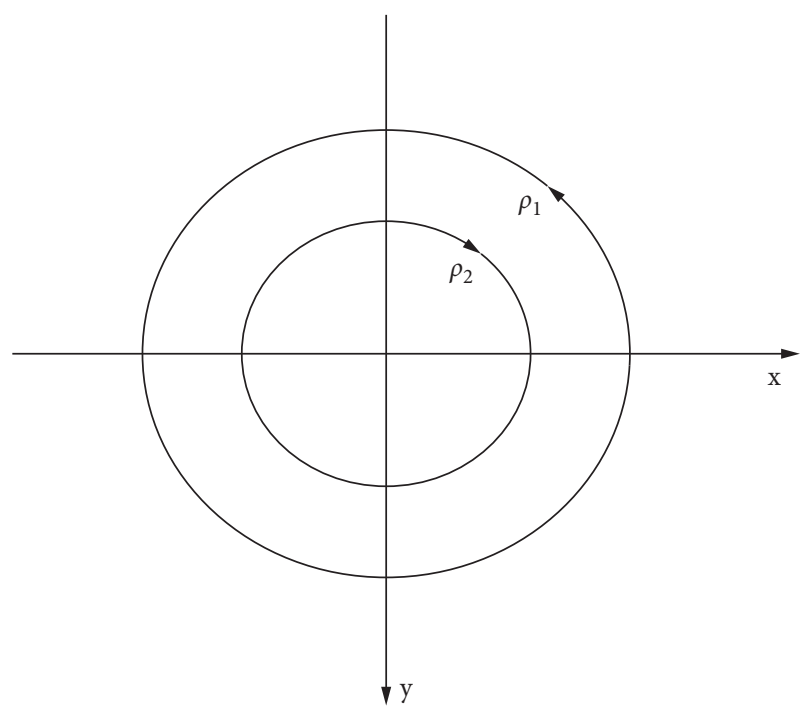

FIGURE 7: Schematic diagram of circular trajectory.

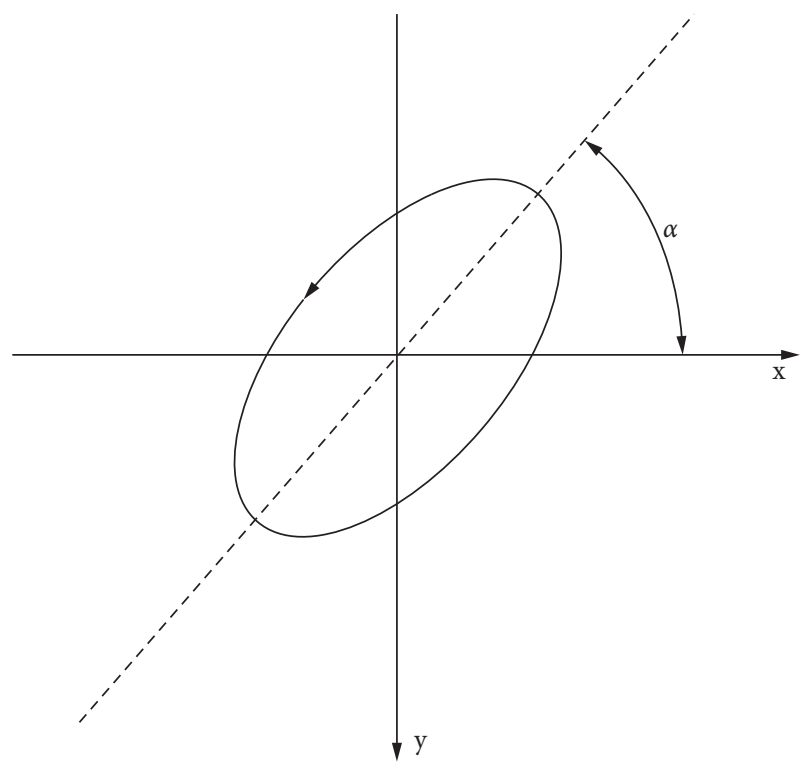

FIgURE 8: Schematic diagram of ellipse motion trajectory.

shaft is measured through two eddy current sensors at $90^{\circ}$ to each other. The installation method of the eddy current sensor during the test is shown in Figure 9. It is particularly important to test the motion state of the rotating shaft when multiple rotating bodies are connected. Due to the complex and diverse delivery methods in the actual process, it is impossible to analyze all the delivery situations one by one. This paper selected the most representative transmission characteristics of the multicylinder transmission mode for testing and analysis. So, the vibration response characteristics and vibration transmission characteristics of the multicylinder parallel chain transmission were studied.

In order to analyze the influence of the vibration coupling of the multicylinder system on the vibration form of the rotating shaft, the tests were mainly divided into two working conditions for comparison, namely, single-cylinder 


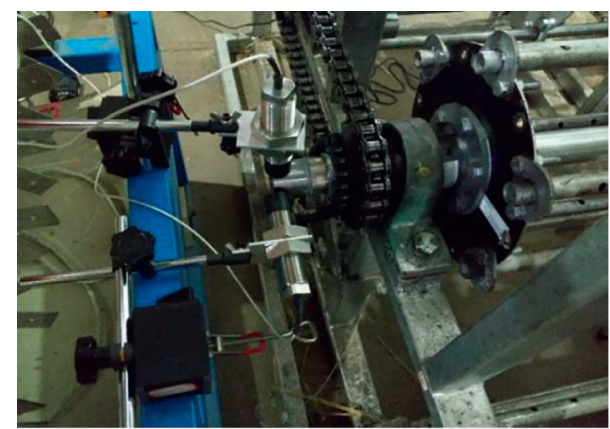

FIgURE 9: Eddy current sensor installation method.

transmission and three-cylinder unilateral transmission. The working conditions in the single-cylinder drive and threecylinder unilateral transmission test were exactly the same. During the tests, an unbalanced mass with a mass of $227.89 \mathrm{~g}$ was applied to the $120^{\circ}$ phase of cylinder I. The shaft of the cylinder I was tested at different rotation speeds. The rotation speed was from $600 \mathrm{r} / \mathrm{min}$ to $800 \mathrm{r} / \mathrm{min}$, each time increasing by $50 \mathrm{r} / \mathrm{min}$.

\section{Results and Discussion}

3.1. Unbalanced Response of Parallel Multicylinders. When there was an unbalanced excitation on a single cylinder, the vibration shape of a single cylinder at $t=0$ is shown in Figure 10. The time-domain imbalance response of each node of a single cylinder is shown in Figure 11.

As shown in Figure 11, the maximum unbalanced response of a single cylinder was at node 2 (excitation position), which was close to the supporting position of the shaft. In addition, there were no supporting unit, corresponding stiffness, and damping at the location of node 3 , so the magnitude of the unbalanced response at this location was relatively large.

The time-domain unbalanced response of each node in a single cylinder is shown in Figure 12. The time-domain signal of each node position was analyzed in the frequency domain to obtain the spectrum diagram shown in Figure 13.

As shown in Figure 12, the frequency of the unbalanced response of each node position is consistent with the frequency conversion. However, due to the presence of damping, the phase of each node position is not the same. Since the damping in the system is not evenly distributed, the phase increments between adjacent nodes are also inconsistent. As shown in Figure 13, the frequency of the unbalanced response was consistent with the frequency of rotation. The difference in the transmission path between each response point and the excitation position led to deviations in the amplitude and phase of each node position.

In order to compare the influence of the transmission system and other working components on the system vibration response, the unbalanced response of the multicylinder unilateral transmission was solved and analyzed. The unbalanced response vibration mode of the system with a multicylinder unilateral transmission is shown in Figure 14.

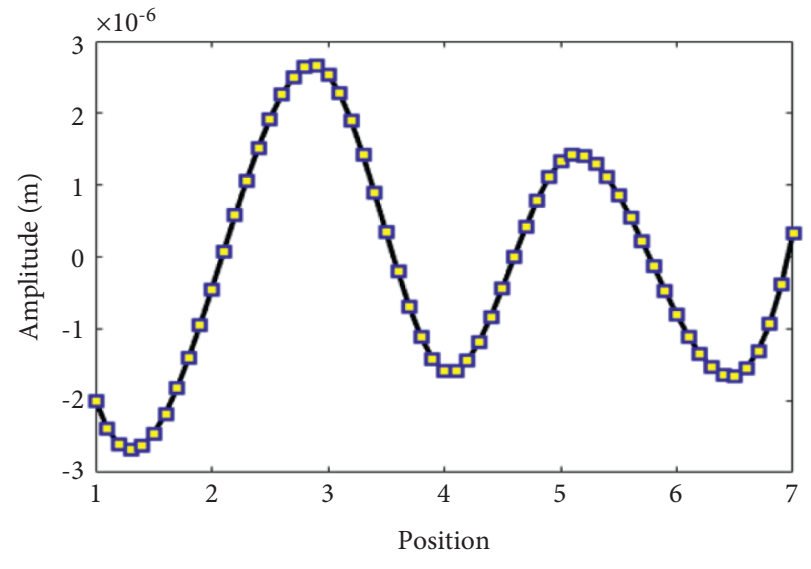

$\square$ Vibration shape of single cylinder at $\mathrm{t}=0$

FIGURE 10: Vibration shape of a single cylinder at $t=0$.

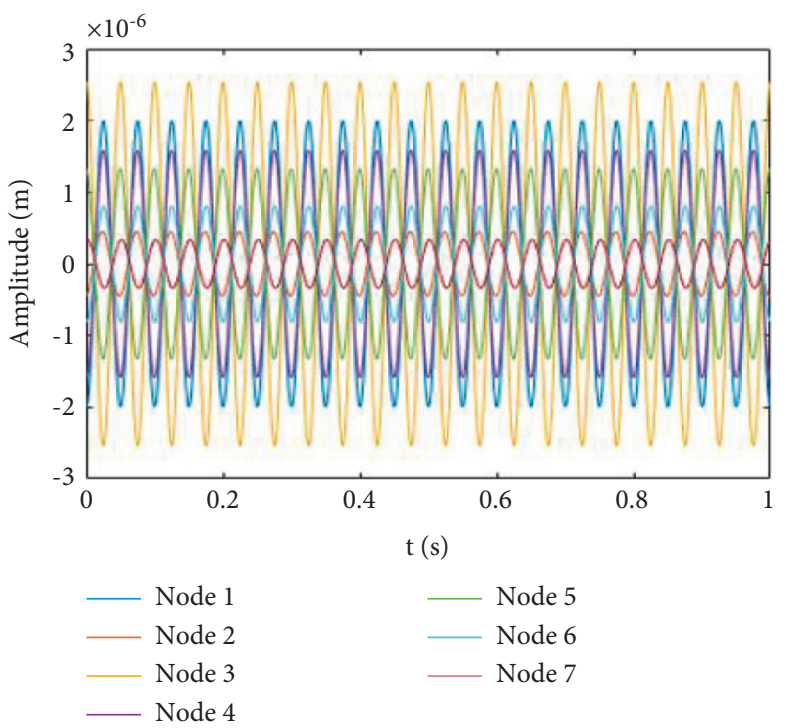

FIgURE 11: Time-domain imbalance response of each node of a single cylinder.

As shown in Figure 14, from the vibration shape of each working part in the multicylinder system, it can be known that the coupling of the transmission system caused the unbalanced response of the entire system to influence each other and overlap each other. There was no unbalanced excitation on the motor shaft, due to the existence of the transmission system. The entire system was connected to each other to form a whole. Due to the unbalanced excitation of other working units in the system, the rotating shaft of the motor also had a corresponding unbalanced response on its rotating shaft. In addition, since the unbalanced excitation on cylinder II was the largest in the actual simulation, its contribution to the actual unbalanced response was also relatively large.

The vibration mode of cylinder I at $t=0$ and the timedomain unbalanced response of each node position in cylinder I in the parallel transmission system are shown in Figures 15 and 16. 


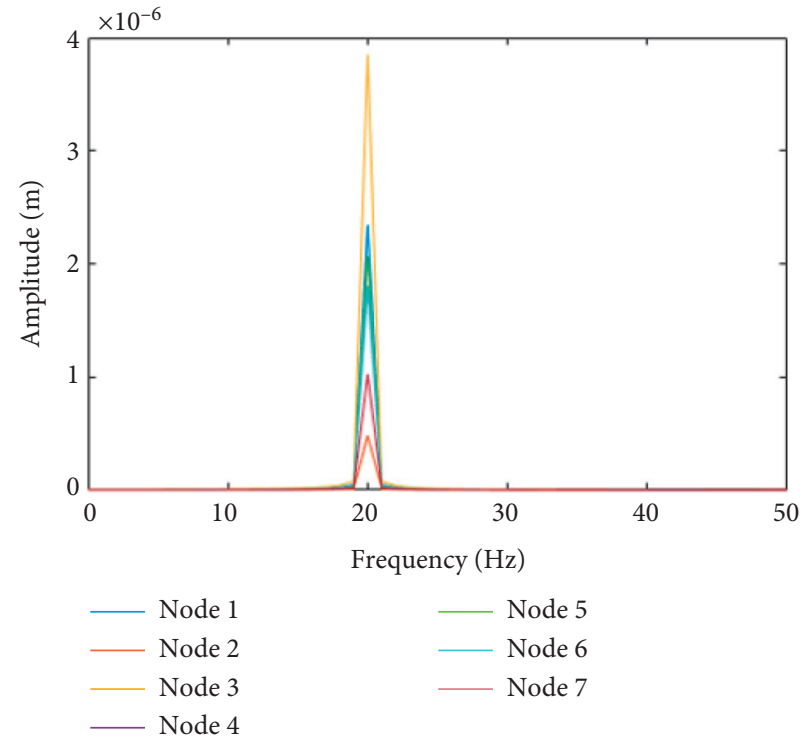

FIGURE 12: Frequency-domain characteristics of a single cylinder.

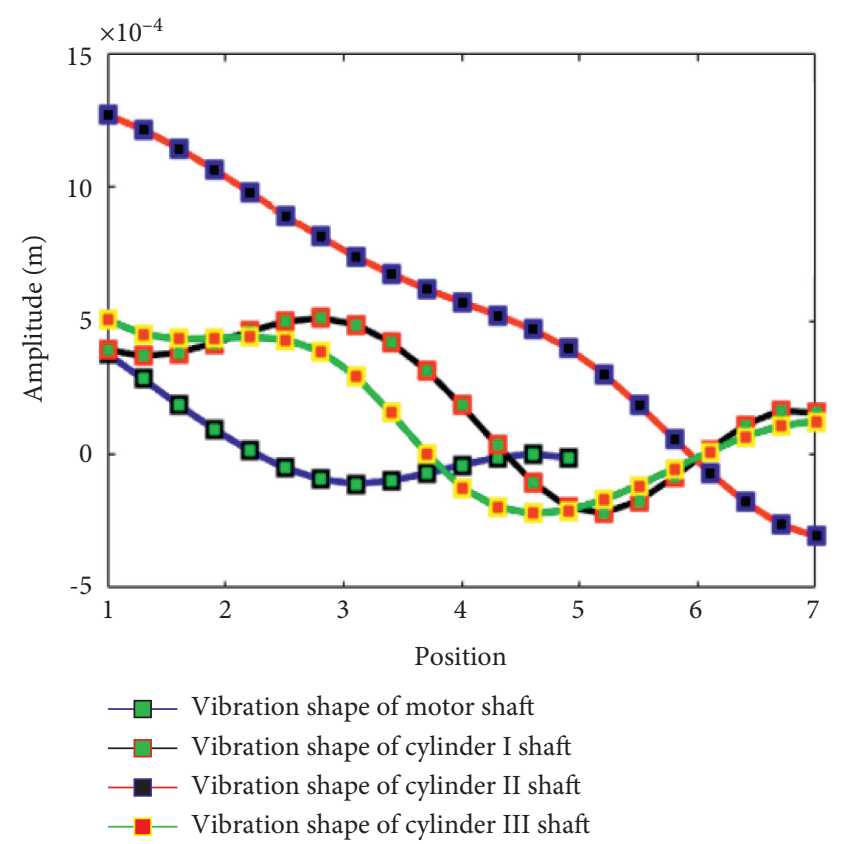

Figure 13: Vibration shape of multicylinder system at $t=0$.

It can be seen from the vibration shape of cylinder I at $t=0$ in Figure 15 that the unbalanced excitation and its own rotation speed were consistent with the single cylinder working alone, but the response generated by the unbalanced excitation was completely different from before. The reason for this phenomenon was that the existence of the transmission system and other working parts caused the boundary conditions of the cylinder I changed. It can be seen from the time-domain unbalanced response of each node position in cylinder I that the time-domain unbalanced response of each node was a periodic signal. It can be seen from the locations of the main peaks on the time-domain

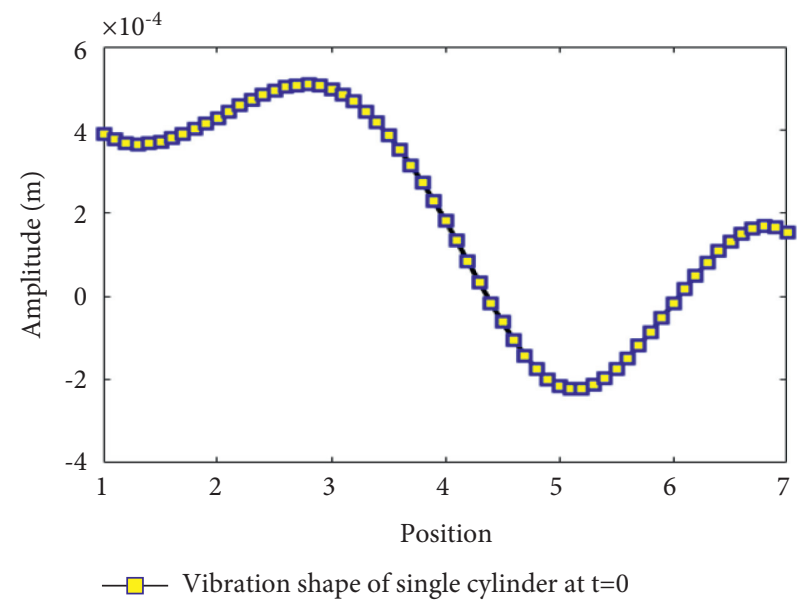

Figure 14: Vibration shape of cylinder I at $t=0$.

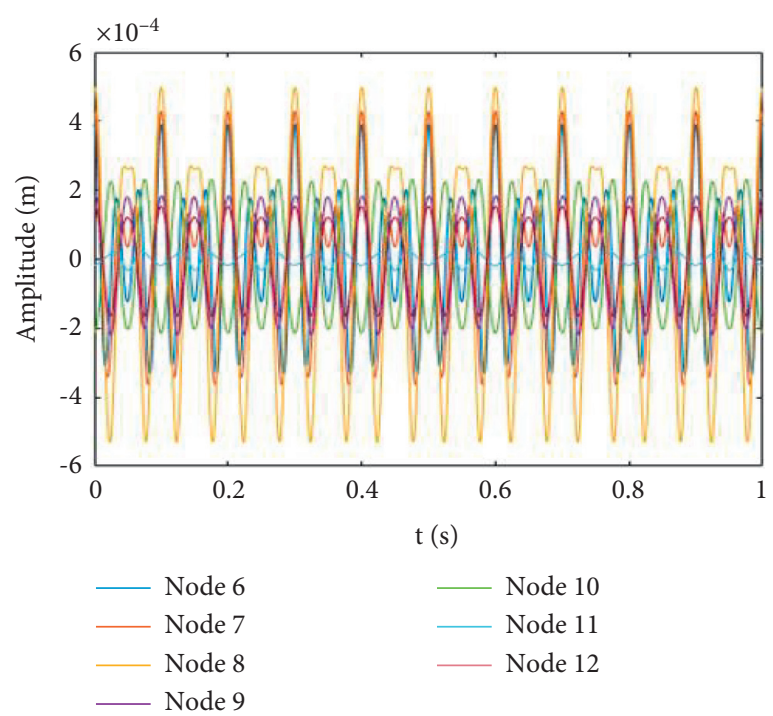

FIGURE 15: Time-domain unbalanced response of each node in cylinder I.

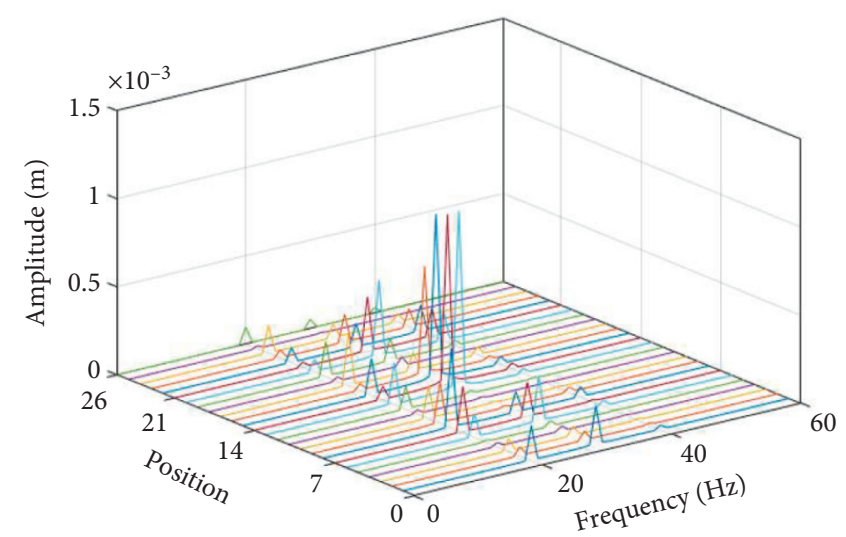

FIGURE 16: Frequency-domain characteristics of multicylinder system. 
unbalanced response that due to the presence of damping, the phase value of the signal had shifted during the transmission process. The vibration response at each node included multiple sine wave components, and finally, these sine components formed a complex curve. In order to further prove this law, the FFT transformation of the parallel multicylinder system obtained the frequency-domain characteristics of the system.

The frequency-domain characteristic of the whole multicylinder system with specific energy is shown in Figure 17.

It can be seen from Figure 17 that the unbalanced response on each rotating body in the system included the vibration component of each working unit. This was mainly because the transmission system was not only used as a boundary condition to change the unbalanced response of the system but also because of its own stiffness and damping; the transmission system also played a role in changing the unbalanced response of the system. Therefore, vibration components of other working parts appeared on the shaft of each rotating unit. As a boundary condition, the transmission system not only affected the vibration response of each working part in the system but also acted as a transmission medium to make the response of each unit in the system influence and superimpose each other.

\subsection{Test Verification for Coupling Characteristics of Parallel Multicylinders}

3.2.1. Vibration Characteristics of Parallel Multicylinders. By testing the vibration response of parallel multicylinder and the vibration state of the rotating shaft under different transmission modes, the influence of chain transmission and different transmission modes on the vibration characteristics of the multicylinder was analyzed.

(1) Vibration Characteristics of Bearing Housing by SingleCylinder Chain Drive. During the working process of the threshing cylinder, the shaft vibration will be transmitted to the bearing seat position through the bearing. Therefore, the vibration response at the bearing seat position can reflect the impact of unbalanced excitation on the system. The unbalanced response of the system is mainly concentrated in the horizontal and vertical directions, so the unbalanced response in the horizontal and vertical directions is mainly measured. When cylinder I was applied with $127.89 \mathrm{~g}$ unbalanced mass on $300^{\circ}$ phase and its rotation speed was $600 \mathrm{r} / \mathrm{min}$, the vibration data of the acceleration sensors in the horizontal direction was taken as an example and analyzed. The FFT results of the vibration acceleration in the range of $0-500 \mathrm{~Hz}$ and $0-30 \mathrm{~Hz}$ are shown in Figure 18 .

As shown in Figure 18, it can be seen that the energy of the bearing housing was larger in the range of 170-200 Hz, of which $170 \mathrm{~Hz}, 180 \mathrm{~Hz}$, and $190 \mathrm{~Hz}$ were the most important energy signals in the entire frequency band. In the actual working process, the fundamental frequency of the threshing cylinder shaft was $10 \mathrm{~Hz}$. The frequency components of this part were the harmonic response of the fundamental frequency, so it had a great relationship with the dynamic imbalance of the shaft. In the range of $0-30 \mathrm{~Hz}$ for a single cylinder, the fundamental frequency of cylinder I and its multiplier were the main frequency components. So the dynamic unbalance was the main reason for the large vibration in the low-frequency range. It can be seen that the vibration of the transmission chain can be transmitted to the cylinder shaft, bearing seat, and frame through the sprocket.

In order to analyze the impact of the transmission chain on the vibration state of the system, the test results of the vibration response of a single-cylinder transmission were sorted and analyzed. The frequency range during analysis was mainly divided into acceleration peaks in the range of $0-30 \mathrm{~Hz}$ and $0-500 \mathrm{~Hz}$. The main data of the vibration acceleration FFT of the bearing seat position under various working conditions is shown in Table 3.

As shown in Table 3, the rotational speed was positively correlated with the unbalanced response of the threshing cylinder at the fundamental frequency. Since cylinder I itself had a certain amount of unbalance, its unbalanced response at the fundamental frequency at the no-load time was not zero. When the unbalanced mass was applied, its response at the fundamental frequency increases significantly. The increase of the speed and the increase of the unbalanced mass on cylinder I made the dynamic unbalance the most important vibration energy of the whole system. In addition, the high-frequency components in the vibration were mostly harmonic responses of the fundamental frequency. The reason for this part of the energy was largely related to the impact and meshing of the transmission chain.

(2) Analysis of Vibration Characteristics of Bearing Housing. In order to verify the influence of multicylinder system on the unbalanced response of the entire system, the vibration characteristics of the multicylinder transmission in parallel were analyzed. It was compared with the result of singlecylinder transmission, so as to further analyze the influence of the system on the unbalanced response of the internal motion unit and compare it with the result of numerical analysis.

The frequency range during analysis was mainly divided into acceleration peaks in the range of $0-30 \mathrm{~Hz}$ and $0-500 \mathrm{~Hz}$. The main data under each working condition are shown in Tables 4 and 5 .

As shown in Tables 4 and 5, as the number of transmission chains in the system increased, the dynamic unbalance response of cylinder I was affected to a certain extent as well. Take the test data of single-cylinder transmission and three-cylinder unilateral transmission as an example for comparative analysis. When the motor speed was $600 \mathrm{r} / \mathrm{min}$ and there was an unbalanced mass on cylinder I, its vibration peaks at the fundamental frequency were $0.107 \mathrm{~m} / \mathrm{s}^{2}$ and $0.096 \mathrm{~m} / \mathrm{s}^{2}$, which were reduced by $10.3 \%$. When the rotation speed was $800 \mathrm{r} / \mathrm{min}$, the vibration peaks at the fundamental frequency were $0.327 \mathrm{~m} / \mathrm{s}^{2}$ and $0.241 \mathrm{~m} / \mathrm{s}^{2}$, which was a decrease of $26.3 \%$. However, when the rotation speed was $750 \mathrm{r} / \mathrm{min}$, the vibration peaks at the fundamental frequency were, respectively, $0.175 \mathrm{~m} / \mathrm{s}^{2}$ and $0.180 \mathrm{~m} / \mathrm{s}^{2}$, an increase of $2.9 \%$. 


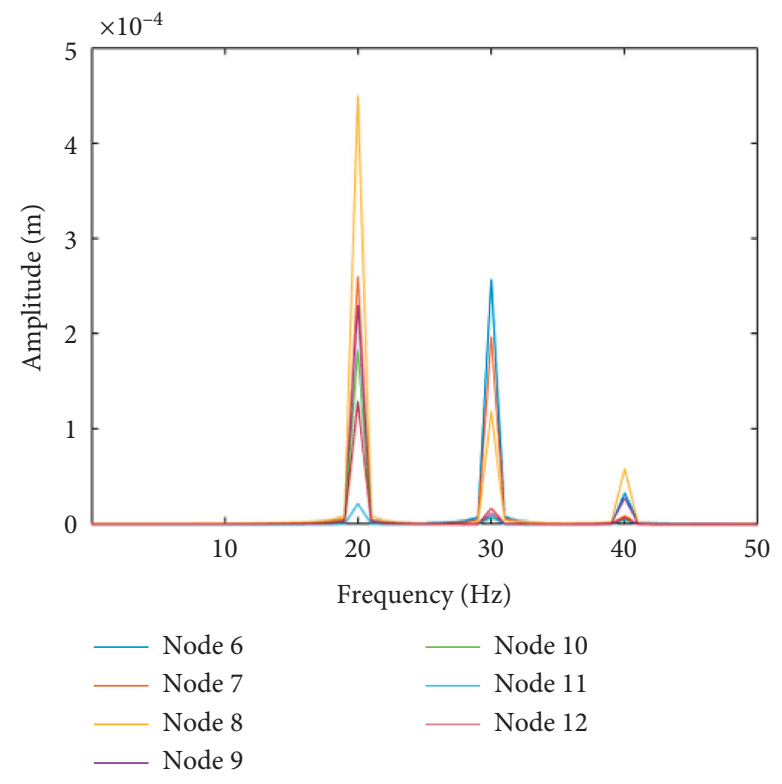

FIgURE 17: Cylinder I frequency-domain characteristics.

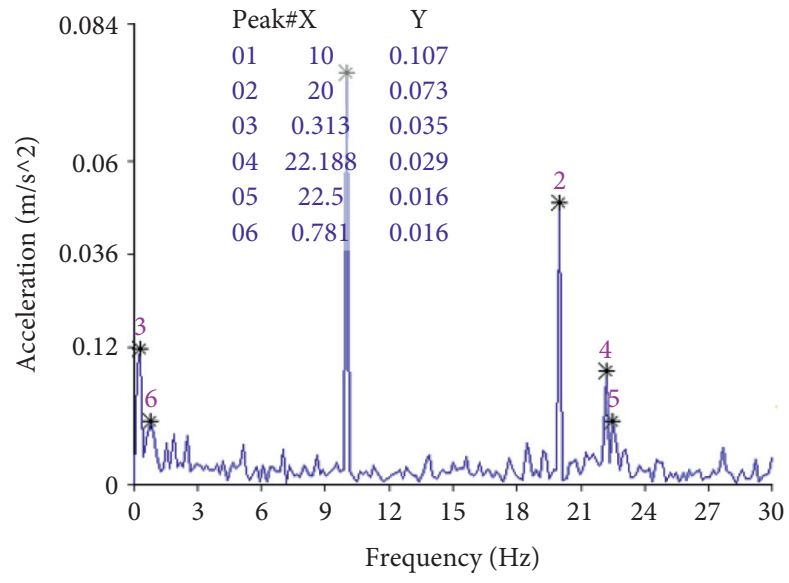

(a)

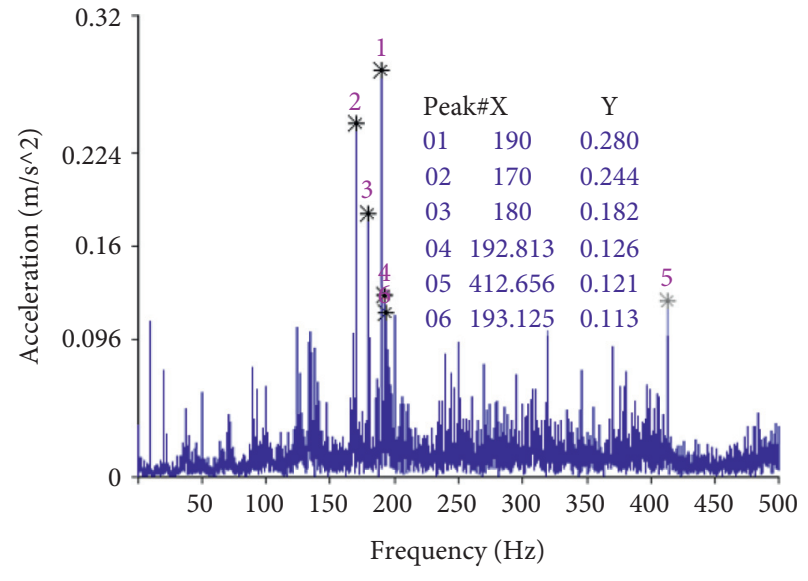

(b)

FIGURE 18: Single-cylinder frequency spectrum in the range of $0-30 \mathrm{~Hz}$ and $0-500 \mathrm{~Hz}$. (a) Spectrogram of single cylinder in the range of $0-30 \mathrm{~Hz}$. (b) Spectrogram of single cylinder in the range of $0-500 \mathrm{~Hz}$.

TABLE 3: FFT main data of vibration acceleration of single-cylinder drive cylinder I at the horizontal position of bearing seat in the $Y$ direction.

\begin{tabular}{|c|c|c|c|c|c|c|c|c|c|}
\hline \multirow{2}{*}{$\begin{array}{l}\text { Rotating speed } \\
(\mathrm{r} / \mathrm{min})\end{array}$} & \multirow{2}{*}{$\begin{array}{l}\text { Frequency } \\
\text { range }(\mathrm{Hz})\end{array}$} & \multicolumn{4}{|c|}{ Single-cylinder drive without load } & \multicolumn{4}{|c|}{ Cylinder I with counterweight $127.89 \mathrm{~g}$} \\
\hline & & $\begin{array}{c}\text { Frequency } 1 \\
(\mathrm{~Hz})\end{array}$ & $\begin{array}{l}\text { Peak 1 } \\
\left(\mathrm{m} / \mathrm{s}^{2}\right)\end{array}$ & $\begin{array}{c}\text { Frequency } 2 \\
(\mathrm{~Hz})\end{array}$ & $\begin{array}{l}\text { Peak 2 } \\
\left(\mathrm{m} / \mathrm{s}^{2}\right)\end{array}$ & $\begin{array}{c}\text { Frequency } 1 \\
(\mathrm{~Hz})\end{array}$ & $\begin{array}{l}\text { Peak 1 } \\
\left(\mathrm{m} / \mathrm{s}^{2}\right)\end{array}$ & $\begin{array}{c}\text { Frequency } 2 \\
(\mathrm{~Hz})\end{array}$ & $\begin{array}{l}\text { Peak } 2 \\
\left(\mathrm{~m} / \mathrm{s}^{2}\right)\end{array}$ \\
\hline \multirow{2}{*}{600} & $0-30$ & 20.00 & 0.074 & 10 & 0.047 & 10.00 & 0.107 & 20.00 & 0.073 \\
\hline & $0-500$ & 190.1 & 0.209 & 170.2 & 0.195 & 190.0 & 0.280 & 170.0 & 0.244 \\
\hline \multirow{2}{*}{650} & $0-30$ & 21.72 & 0.185 & 10.78 & 0.040 & 21.72 & 0.161 & 10.78 & 0.119 \\
\hline & $0-500$ & 401.2 & 0.268 & 195.2 & 0.240 & 195.0 & 0.235 & 400.1 & 0.229 \\
\hline \multirow{2}{*}{700} & $0-30$ & 11.72 & 0.059 & 22.34 & 0.047 & 11.72 & 0.175 & 21.56 & 0.042 \\
\hline & $0-500$ & 198.4 & 0.269 & 408.4 & 0.171 & 198.4 & 0.211 & 210.0 & 0.187 \\
\hline \multirow{2}{*}{750} & $0-30$ & 12.50 & 0.098 & 22.19 & 0.036 & 12.50 & 0.295 & 22.00 & 0.168 \\
\hline & $0-500$ & 237.7 & 0.380 & 219.0 & 0.294 & 237.3 & 0.325 & 12.50 & 0.295 \\
\hline \multirow{2}{*}{800} & $0-30$ & 13.28 & 0.145 & 26.56 & 0.078 & 26.56 & 0.363 & 13.28 & 0.327 \\
\hline & $0-500$ & 239.7 & 0.690 & 253.0 & 0.416 & 240.0 & 0.676 & 253.3 & 0.425 \\
\hline
\end{tabular}


TABLE 4: FFT main data of vibration acceleration of three-cylinder unilateral drive cylinder I in the horizontal position of the bearing seat in the $Y$ direction.

\begin{tabular}{lccccccccc}
\hline $\begin{array}{l}\text { Rotating speed } \\
(\mathrm{r} / \mathrm{min})\end{array}$ & $\begin{array}{c}\text { Frequency } \\
\text { range }(\mathrm{Hz})\end{array}$ & $\begin{array}{c}\text { Cylinder I with counterweight 127.89 g } \\
(\mathrm{Hz})\end{array}$ & $\begin{array}{c}\text { Peak 1 } \\
\left(\mathrm{m} / \mathrm{s}^{2}\right)\end{array}$ & $\begin{array}{c}\text { Frequency 2 } \\
(\mathrm{Hz})\end{array}$ & $\begin{array}{c}\text { Peak 2 } \\
\left(\mathrm{m} / \mathrm{s}^{2}\right)\end{array}$ & $\begin{array}{c}\text { Frequency 1 } \\
(\mathrm{Hz})\end{array}$ & $\begin{array}{c}\text { Peak 1 } \\
\left(\mathrm{m} / \mathrm{s}^{2}\right)\end{array}$ & $\begin{array}{c}\text { Frequency 2 } \\
(\mathrm{Hz})\end{array}$ & $\begin{array}{l}\text { Peak 2 } \\
\left(\mathrm{m} / \mathrm{s}^{2}\right)\end{array}$ \\
\hline \multirow{2}{*}{600} & $0-30$ & 22.34 & 0.123 & 10.00 & 0.096 & 22.34 & 0.180 & 10.00 & 0.034 \\
& $0-500$ & 180.3 & 0.737 & 194.7 & 0.622 & 180.0 & 1.051 & 187.2 & 0.924 \\
\hline \multirow{2}{*}{650} & $0-30$ & 21.72 & 0.126 & 10.94 & 0.102 & 21.72 & 0.158 & 10.78 & 0.038 \\
& $0-500$ & 203.4 & 0.883 & 195.6 & 0.876 & 195.2 & 0.745 & 203.0 & 0.597 \\
\hline \multirow{2}{*}{700} & $0-30$ & 11.72 & 0.180 & 23.44 & 0.124 & 25.16 & 0.091 & 11.72 & 0.052 \\
& $0-500$ & 210.5 & 0.899 & 218.9 & 0.668 & 209.8 & 0.724 & 218.2 & 0.803 \\
\hline \multirow{2}{*}{750} & $0-30$ & 12.50 & 0.256 & 25.00 & 0.134 & 12.50 & 0.092 & 21.87 & 0.069 \\
& $0-500$ & 233.8 & 0.559 & 225.0 & 0.434 & 225.3 & 0.615 & 234.4 & 0.567 \\
\hline \multirow{2}{*}{800} & $0-30$ & 13.28 & 0.241 & 26.64 & 0.181 & 13.28 & 0.105 & 22.97 & 0.086 \\
& $0-500$ & 250.0 & 0.852 & 230.7 & 0.696 & 249.8 & 1.062 & 230.6 & 0.571 \\
\hline
\end{tabular}

TABLE 5: FFT main data of vibration acceleration of three-cylinder bilateral drive cylinder I in the horizontal position of the bearing seat in the $Y$ direction.

\begin{tabular}{lccccccccc}
\hline $\begin{array}{l}\text { Rotating speed } \\
(\mathrm{r} / \mathrm{min})\end{array}$ & $\begin{array}{c}\text { Frequency } \\
\text { range }(\mathrm{Hz})\end{array}$ & $\begin{array}{c}\text { Cylinder I with counterweight 127.89g } \\
(\mathrm{Hz})\end{array}$ & $\begin{array}{c}\text { Peak 1 } \\
\left(\mathrm{m} / \mathrm{s}^{2}\right)\end{array}$ & $\begin{array}{c}\text { Frequency 2 } \\
(\mathrm{Hz})\end{array}$ & $\begin{array}{c}\text { Peak 2 } \\
\left(\mathrm{m} / \mathrm{s}^{2}\right)\end{array}$ & $\begin{array}{c}\text { Frequency 1 } \\
(\mathrm{Hz})\end{array}$ & $\begin{array}{c}\text { Peak 1 } \\
\left(\mathrm{m} / \mathrm{s}^{2}\right)\end{array}$ & $\begin{array}{c}\text { Frequency 2 } \\
(\mathrm{Hz})\end{array}$ & $\begin{array}{l}\text { Peak 2 } \\
\left(\mathrm{m} / \mathrm{s}^{2}\right)\end{array}$ \\
\hline \multirow{2}{*}{600} & $0-30$ & 22.17 & 0.142 & 10.00 & 0.106 & 22.28 & 0.047 & 10.00 & 0.035 \\
& $0-500$ & 180.12 & 0.394 & 187.3 & 0.283 & 180.0 & 0.375 & 187.2 & 0.369 \\
\hline \multirow{2}{*}{650} & $0-30$ & 21.67 & 0.154 & 10.80 & 0.098 & 21.67 & 0.125 & 10.80 & 0.047 \\
& $0-500$ & 97.47 & 0.476 & 202.8 & 0.334 & 202.6 & 0.405 & 97.40 & 0.331 \\
\hline \multirow{2}{*}{700} & $0-30$ & 11.66 & 0.195 & 25.21 & 0.112 & 25.27 & 0.079 & 11.66 & 0.045 \\
& $0-500$ & 210.0 & 0.344 & 198.4 & 0.294 & 210.3 & 0.328 & 201.9 & 0.287 \\
\hline \multirow{2}{*}{750} & $0-30$ & 12.51 & 0.279 & 25.02 & 0.167 & 12.51 & 0.081 & 21.54 & 0.058 \\
& $0-500$ & 112.6 & 0.228 & 225.3 & 0.226 & 225.5 & 0.332 & 112.7 & 0.313 \\
\hline \multirow{2}{*}{800} & $0-30$ & 13.31 & 0.258 & 22.95 & 0.208 & 13.37 & 0.125 & 221.10 & 0.091 \\
& $0-500$ & 240.0 & 0.740 & 226.7 & 0.401 & 240.5 & 0.627 & 182.7 & 0.548 \\
\hline
\end{tabular}

It can be found that the existence of the transmission chain had a more complicated influence on the vibration response of the system, which was not only related to the unbalance of the cylinder itself but also had a great relationship with the speed. As shown in Tables 4 and 5, it can be seen that not only will the existence of the transmission chain itself affect the vibration state of the system, but also the change of the transmission mode will cause the state of the system to change. Under the different transmission modes, the unbalanced response of each working part in the multicylinder system at the fundamental frequency had changed to a certain extent, and the overall change was within $30 \%$. From the theoretical calculation and analysis of the multicylinder system, it can be seen that the existence of the transmission chain, the change of the transmission mode, and the existence of other working components will change the inherent characteristics of the entire multicylinder system and eventually lead to corresponding differences in the response of the system.

3.2.2. Trajectory of Rotating Shaft Undergoing Vibration Coupling. For parallel multicylinder systems, the axis trajectory is not only related to the motion state but also affected by the transmission mode and support. The main transmission mode of the parallel multicylinder system is chain drive or belt drive. Take the chain drive as an example for analysis. It is connected to the shaft of the threshing cylinder through a sprocket during work. When it is working, the tight side of the chain drive can be equivalent to a continuous rod with a certain rigidity but less damping. This may cause the vibration at the fundamental frequency of the adjacent cylinders to be transmitted to the rotating shaft through the transmission chain, thereby superimposing with the forced vibration of the rotating shaft itself. Due to the rigidity of the frame and better transmission, the vibration components of adjacent cylinders may also be transmitted to the rotating shaft through the frame and then through the bearing seat. Considering that there is a certain working gap during the reworking process of the bearing, the relative vibration transmission amount of this part is relatively small.

Based on the above situation, it can be seen that without considering other failures of the threshing cylinder, the vortex shape of the rotating shaft of the parallel multicylinder system is not completely elliptical. It will inevitably be mixed with other frequency components in the working process, which causes its vortex trajectory to be closer to the 


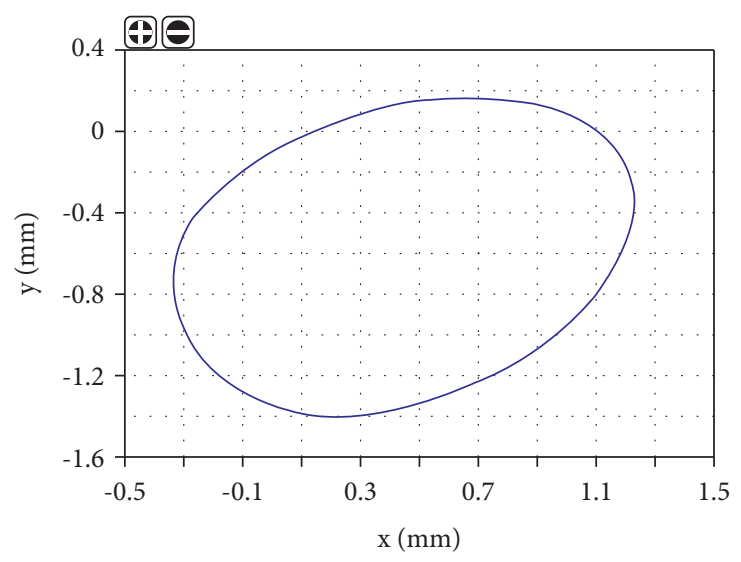

Figure 19: Test axis trajectory.

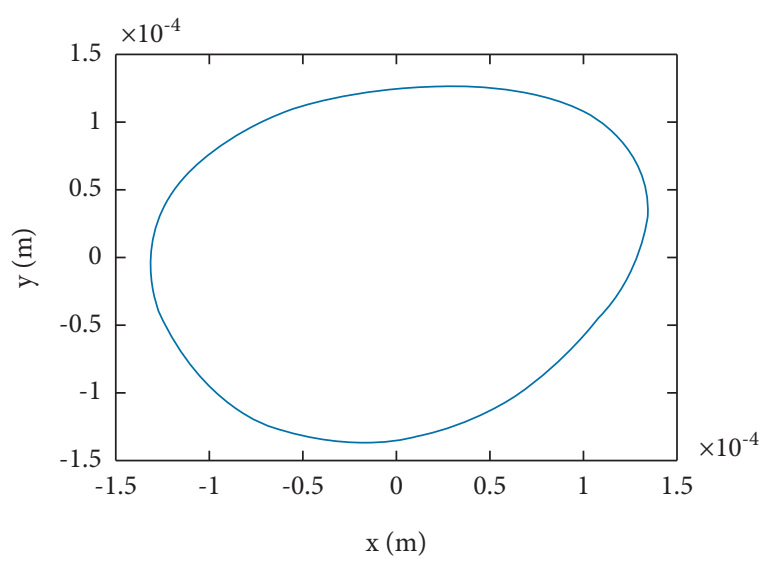

FIgURE 20: Theoretical vortex trajectory.

"duck egg shape"; that is, one end is larger and the other is smaller. The specific shape characteristics are closely related to the transmission characteristics of the system. In this paper, this kind of situation also appeared in the test process. Taking the axis trajectory of cylinder I when the three cylinders were transmitted on one side as an example, the measured shape during the test is shown in Figure 19.

As shown in Figure 19, when cylinder I was connected to other threshing cylinders with different speeds, it can be seen from the axis track that the vortex shape of the shaft end was not a standard ellipse. In order to further explain when the axis track of the rotating shaft contains the frequency components of the adjacent cylinders, the vortex shape will change to a certain extent. The simulation and comparative analysis of three-cylinder unilateral transmission was carried out. The main parameters of the simulation, including the equivalent stiffness of the transmission chain and the support stiffness of the bearing seat, were the same as the data used in the previous simulation. The vibration response in the $x$ and $y$ directions at the node 6 position of cylinder I close to the bearing and the transmission chain position was superimposed and solved.

The theoretical whirl trajectory at the node 6 position was obtained, as shown in Figure 20. It can be seen that the shape of the shaft center trajectory obtained by simulation and the measured shaft center trajectory had very similar characteristics. Neither of them can be completely regarded as the "elliptical" vortex shape and was closer to the "duck egg shape". From the perspective of theoretical analysis, it was mainly caused by the transmission characteristics of the system. Since the main vibration components of adjacent cylinders at the fundamental frequency will be transmitted and superimposed through a specific transmission path, the vortex shape of the rotating shaft will change to a certain extent.

\section{Conclusion}

(1) According to the composition of the general expression of the unbalanced response of the multicylinder system including the transmission system, it can be known that the existence of the transmission system and the change of the transmission mode will change the boundary conditions of the system. The existence of the transmission system essentially changed the mass, stiffness, and the value and position of the elements in the damping matrix of the system, which ultimately changed the unbalanced response of the multicylinder system.

(2) Through the numerical simulation of the multicylinder system and the experimental verification, the existence of the transmission system and the change of the transmission method will cause the unbalanced response of the entire system to change. The contribution rate of the influence of different transmission methods and speeds to the vibration amount at the fundamental frequency of the multicylinder was $5 \%$ to $30 \%$.

(3) In view of the comparative analysis of actual test and simulation results, it can be known that the existence of the transmission system led to a certain change in the motion law of the rotating shaft. The vortex shape of the rotating shaft changed from a standard "ellipse" to a "duck egg" vortex shape containing multiple excitation frequencies. This proved from the side that the vibration coupling of the transmission system changed the motion state of the rotating shaft and the unbalanced response of the system as well. 


\section{Data Availability}

The data used to support the findings of this study are available from the corresponding author upon request.

\section{Conflicts of Interest}

The authors declare that they have no conflicts of interest.

\section{Acknowledgments}

This research work was supported by the National Natural Science Foundation of China (52175235), Single Technology Research and Development Project of Jiangsu Agricultural Science and Technology Innovation Fund (CX(21)3144), Jiangsu Province Research and Practice Innovation Program Project (KYCX21_3382), and Key Laboratory of Crop Harvesting Equipment Technology of Zhejiang Province (2021KY02).

\section{References}

[1] Z. Tang, Y. Li, X. Li, and T. Xu, "Structural damage modes for rice stalks undergoing threshing," Biosystems Engineering, vol. 186, pp. 323-336, 2019.

[2] Z. Tang, Y. Q. Liang, Y. Li et al., "Effects of multi-sequence combination forces on creep characteristics of bales during wheat harvesting," International Journal of Agricultural and Biological Engineering, vol. 14, pp. 88-99, 2021.

[3] Z. Tang, B. Zhang, M. L. Wang, and H. T. Zhang, "Damping behaviour of a prestressed composite beam designed for the thresher of a combine harvester," Biosystems Engineering, vol. 204, pp. 130-146, 2021.

[4] P. F. Wang, J. J. Zhang, and D. Y. Chen, "Hamiltonian modeling and dynamic analysis of shafting system in hydroturbine generating set," Journal of Drainage and Irrigation Machinery Engineering, vol. 38, no. 8, pp. 794-800, 2020.

[5] Z. Tang, X. Y. Li, X. D. Chen, and Y. Chen, "Design of negative pressure spiral feeding device for tangential and longitudinal axial intersection of combine harvester," Advances in Materials Science and Engineering, Art, vol. 2019, Article ID 1647230, 12 pages, 2019.

[6] K. Yoshida and W. Khalil, "A new model for dynamic behaviour of timing belt drive systems," IFAC Proceedings Volumes, vol. 33, no. 26, pp. 631-636, 2000.

[7] A. Ward and R. S. Dwyer-Joyce, "Model experiments on automotive chain drive systems," Tribology Series, vol. 39, pp. 851-861, 2001.

[8] O. A. Assad, E. Godoy, and V. Croulard, "Macroscopic drive chain efficiency modeling using state machines," IFAC Proceedings Volumes, vol. 41, no. 2, pp. 2294-2299, 2008.

[9] Y. Wang, D. S. Ji, and K. Zhan, "Modified sprocket tooth profile of roller chain drives," Mechanism and Machine Theory, vol. 70, pp. 380-393, 2013.

[10] G. Čepon and M. Boltežar, "Dynamics of a belt-drive system using a linear complementarity problem for the belt-pulley contact description," Journal of Sound and Vibration, vol. 319, no. 3-5, pp. 1019-1035, 2009.

[11] G. Čepon, L. Manin, and M. Boltežar, "Introduction of damping into the flexible multibody belt-drive model: a numerical and experimental investigation," Journal of Sound and Vibration, vol. 324, no. 1-2, pp. 283-296, 2009.
[12] C. Pereira, J. Ambrósio, and A. Ramalho, "Dynamics of chain drives using a generalized revolute clearance joint formulation," Mechanism and Machine Theory, vol. 92, pp. 64-85, 2015.

[13] Y. M. Li, H. R. Cao, and K. Tang, “A general dynamic model coupled with EFEM and DBM of rolling bearing-rotor system," Mechanical Systems And Signal Processing, vol. 134, Article ID 106322, 2019.

[14] P. Kumar and R. Tiwari, "Finite element modelling, analysis and identification using novel trial misalignment approach in an unbalanced and misaligned flexible rotor system levitated by active magnetic bearings," Mechanical Systems and Signal Processing, vol. 152, Article ID 107454, 2021.

[15] O. T. Filsoof, M. H. Hansen, A. Yde, P. Bttcher, and X. Zhang, "A novel methodology for analyzing modal dynamics of multi-rotor wind turbines," Journal of Sound and Vibration, vol. 493, pp. 1-8, 2021.

[16] A. Mereles and K. L. Cavalca, "Modeling of multi-stepped rotor-bearing systems by the continuous segment method," Applied Mathematical Modelling, vol. 96, pp. 402-430, 2021.

[17] A. Mereles and K. L. Cavalca, "Mathematical modeling of continuous multi-stepped rotor-bearing systems," Applied Mathematical Modelling, vol. 90, pp. 327-350, 2021.

[18] R. Dupont, "Robust rotor dynamics for high-speed air bearing spindles," Precision Engineering, vol. 40, pp. 7-13, 2015.

[19] J. Z. Vance, F. Murphy, and Brian, "Machinery vibration and rotordynamics," Wiley, Hoboken, NJ, USA, 2010.

[20] E. Chipato, A. D. Shaw, and M. I. Friswell, "Frictional effects on the nonlinear dynamics of an overhung rotor," Communications in Nonlinear Science and Numerical Simulation, vol. 78, pp. 1-12, 2019.

[21] Y. Q. Li, Z. Luo, J. X. Liu, H. Ma, and D. S. Yang, "Dynamic modeling and stability analysis of a rotor-bearing system with bolted-disk joint," Mechanical Systems and Signal Processing, vol. 158, Article ID 107778, 2021.

[22] H. C. Cheng, Y. M. Zhang, W. J. Lu, and Z. Yang, "Mechanical characteristics and nonlinear dynamic response analysis of rotor-bearing-coupling system," Applied Mathematical Modelling, vol. 93, pp. 708-727, 2021.

[23] Y. J. Chiu and D. Z. Chen, "The coupled vibration in a rotating multi-disk rotor system," International Journal of Mechanical Sciences, vol. 53, no. 1, pp. 1-10, 2011.

[24] G. Y. Zhang, F. Ji, W. G. Zhao, and T. J. Li, "Study on the collaborative design PN-pddp model for the multi-component coupling rotor system based on petri nets," Procedia CIRP, vol. 56, pp. 67-72, 2016.

[25] Z. W. Mu, X. W. Gui, Q. C. Xia, and Z. S. Zhang, "Structural optimization design of impeller vane of shaft seal pump based on CFD," Journal of Drainage and Irrigation Machinery Engineering, vol. 39, no. 1, pp. 1-7, 2021.

[26] H. Quan, J. Cheng, and G. Y. Peng, "Effect of screw centrifugal inducer on mechanical characteristics of vortex pump," Journal of Drainage and Irrigation Machinery Engineering, vol. 39, no. 4, pp. 345-350, 2021.

[27] S. R. Chen, Y. P. Zhou, Z. Tang, and S. N. Lu, "Modal vibration response of rice combine harvester frame under multi-source excitation," Biosystems Engineering, vol. 194, pp. 176-195, 2020. 